\title{
Short-term forecasting for empirical economists. A survey of the recently proposed algorithms
}

\author{
Maximo Camacho \\ Universidad de Murcia \\ mcamacho@um.es
}

\author{
Gabriel Perez-Quiros* \\ Banco de España and CEPR \\ gabriel.perez@bde.es
}

Pilar Poncela

Universidad Autónoma de Madrid

pilar.poncela@uam.es

\begin{abstract}
Practitioners do not always use research findings, sometimes because the research is not always conducted in a manner relevant to real-world practice. This survey seeks to close the gap between research and practice on short-term forecasting in real time. Towards this end, we review the most relevant recent contributions to the literature, examine their pros and cons, and we take the liberty of proposing some lines of future research. We include bridge equations, MIDAS, VARs, factor models and Markovswitching factor models, all allowing for mixed-frequency and ragged ends. Using the four constituent monthly series of the Stock-Watson coincident index, industrial production, employment, income and sales, we evaluate their empirical performance to forecast quarterly US GDP growth rates in real time. Finally, we review the main results regarding the number of predictors in factor based forecasts and how the selection of the more informative or representative variables can be made.
\end{abstract}

Keywords: Business Cycles, Output Growth, Time Series.

JEL Classification: E32, C22, E27

\footnotetext{
${ }^{*}$ Corresponding Author: Gabriel Perez Quiros, Banco de España, Research Division, Monetary and Financial Studies Department, DG Economics, Statistics, and Research. Alcalá 48, 28014 Madrid, Spain. E-mail: gabriel.perez@bde.es. M. Camacho and P. Poncela acknowledges support from projects ECO2010-19830, ECO2009-10287 and ECO2012-32854, respectively.
} 


\section{Introduction}

The rapid economic changes during the Great Recession were a big shock to policy makers and the business world. In 2008, the sharp downturn in the economy triggered drastic reactions by policy makers who implemented monetary and fiscal policies to combat the adverse economic situation. In addition, the pervasive effects on retirement plans, stock portfolios and the housing market drastically changed private agents' economic decisions. Since being late in identifying the turning points entailed dramatic economic consequences, the economic agents seemed eager to learn as quickly as possible early detection methods to foresee downturns and recoveries and they acknowledged the need for new tools to monitor economic developments in real time. These forecasts usually include predictions of changes in the gross domestic product (GDP) and its components, both from an expenditure perspective (consumption, gross capital formation, exports and imports) and from a revenue perspective. Sometimes, they also deal with the revenue and expenditure flows between the different institutional sectors, inflation, the labour market and financial data.

Consequently, business people all over the world seem to have caught gold fever to speedily update the next relevant macroeconomic figures with significant time to onset. Accurate forecasts of these figures allow them to position themselves competitively since advance notice gives the business time to implement new strategies. In this survey, we focus on several statistical frameworks developed in the literature to perform early assessments of the ongoing economic development, which must unavoidably deal with short-term forecasting. According to this scenario planning, the time period attributed in this paper to examine the accuracy of short-term forecasts goes from the publication of the latest available figure of one variable of interest to the availability of the next figure. Actually, this time period concentrates the forecasting interest of business people in real time, in what it is called nowcasting, which rarely covers more than a few months.

As a response to this growing interest, this paper is written as a survey that aims to close the potential gap between research and applied short-term forecasting. We establish some of the key theoretical results and empirical findings in the recent literature on shortterm forecasting. Then, we try to translate these theoretical findings into economically 
meaningful techniques to facilitate their widespread application to compute short-term forecast in economics and to monitor the ongoing business cycle developments in real time. In this sense, the survey does not pretend to be a textbook in forecasting, covering all the aspects of the forecasting exercise, from theoretical definitions of predictability to the definition of loss function for the forecasting exercise. For an excellent survey on the definition of forecasting predictability, Hendry and Mizon (2012) is highly recommended. For a comprehensive guide to forecasting, the books of Clements and Hendry (1998) or Diebold (1998) are excellent references.

It is worth noting that the automatic forecasting frameworks surveyed in this paper exhibit several advantages with respect to the economic forecasts typically provided by the most relevant economic institutions. Although these institutions usually employ state-ofthe-art forecasting methods, they have the possibility of partly basing their forecasts on judgements. Consequently, their forecasts cannot be easily replicated and their forecast failures are difficult to interpret. By contrast, the statistical methods described in this survey seek to avoid this problem by using simple forecasting algorithms which, while doing the job of computing short-term forecasts, have the advantage of forecasting from specific frameworks. Therefore, the methods can be evaluated in terms of transparency and replicability. In addition, the automatic forecasts can be easily updated as new releases for the key economic indicators become available. This considerably reduces the time to process the economic information because it is done as new data arrive and this quick processing facilitates rapid reactions to economic news.

However, computing the short-term forecasts in real time is not straightforward. To start with, the analysts performing multiperiod forecasting must choose between either using a one-period model that is iterated forward, or instead a multi-period model estimated with a loss function tailored to the forecast horizon. Although the iterated method produces more efficient parameter estimates than the direct method and does not require different models for different forecasting horizons, it is prone to bias if the one-step-ahead model is misspecified and usually requires separate forecasting models for the explanatory variables. Which approach is better will depend on the characteristics of the forecasting model and, ultimately, will be an empirical matter. 
In addition, performing short-term forecasts in real time faces specific problems of the day-to-day monitoring of economic developments. The first problem is that the real-time data flow of all the variables involved in the forecasting analysis does not occur at the same time. Although the national statistical agencies release economic data in blocks and the releases follow a relatively stable calendar, most of the releases are asynchronous. Moreover, the economic indicators of the current state of the economy are available with different delays. Typically, hard indicators, which refer to economic activity data, exhibit relatively long reporting lags, which usually extend to two months. Soft indicators, which are based on opinion surveys, are released on a more timely basis since they are usually available at the end of the reference month. Financial data are available on a daily basis and are also available at the end of the month. According to this particular release process, the automatic forecasting models should support reading linked data in an asynchronous manner.

Not accounting for this publication pattern would imply that the users of traditional forecasting models, which develop the forecasts from balanced panels of data, will unavoidably incur one of the two following substantial costs. The first appears when forecasts are made from the latest available balanced panel. In this case, the forecasts lose the latest and most valuable information contained in the promptly issued indicators at the time of the assessments. The second cost is that of being late. If the analysts decide to wait until all the business cycle indicators become available, their inference will refer to the past.

The second problem of real-time short-term forecasting is that it usually involves time series data sampled at different frequencies. Many important macroeconomic indicators, which are the key time series to be predicted, are sampled at low frequency and are published with a significant delay. One noticeable example is GDP, whose data are sampled quarterly and are released with a delay of about one and a half months with respect to the end of the reference quarter. With the aim of obtaining early estimates of these lowfrequency variables, the analysts frequently focus on higher-frequency economic indicators. These indicators typically show high correlation with the low-frequency variables but exhibit much more timely information at monthly, weekly, daily, or even higher frequencies.

The earliest attempts to assess the peculiarities of real-time forecasting were based 
on bridge equation models. Essentially, these are single-equation autoregressive models that focus on the lower-frequency variable of interest which is modeled as a function of aggregations of the higher-frequency economic indicators. To compute the forecasts, the higher-frequency indicators are usually predicted in separate autoregressive models. Although bridge equations usually incur parameter proliferation problems, they are very popular in Central Banks and research institutions because of their simplicity and modest technical requirements.

With parsimony in mind, MIDAS models are general frameworks that require a small number of hyperparameters relative to the sampling rate of the higher-frequency. Typically, they employ distributed lag polynomials as weighting functions of lagged values of the higher-frequency indicators, which require nonlinear estimation techniques. Since they are relatively new on the forecasting arena, they are a fruitful place to consider further developments. In this paper, we review the latest research and take the liberty of suggesting future lines on this topic.

Some other proposals assume that the models operate at the highest frequency in the data, which implies that some values of the lower-frequency data and the latest data of the indicators with longer publication delays are unobserved. The models are conveniently cast in state-space representations and are estimated by using the Kalman filter since it has the ability to account for missing observations in a data set in a relatively straightforward manner. In short, the strategy consists of skipping some calculations while others do not need to be changed, so the basic Kalman filter remains valid and the parameters of the model can be estimated by maximum likelihood. This feature is of practical relevance when computing the forecasts since one can regard the future values of the time series as a set of missing observations. As a consequence, the Kalman also delivers the necessary computations for forecasting. Given the population parameters, the Kalman filter also provides the Mean Square Forecast Error (MSFE). In addition, these models have been extended to account for regime-switching nonlinearities. They are used to infer the probabilities of recession that serve as a barometer for the state of the business cycle.

Practitioners usually compute the MSFEs as if their estimated parameters were the "true" ones. Therefore, they do not take into account the uncertainty associated to the 
estimation of the parameters, and their prediction intervals are usually narrower than they should be. To overcome this problem, Wall and Stoffer (2002), Pfeffermann and Tiller (2005) and Rodríguez and Ruiz (2009) propose using bootstrap techniques to compute mean square errors (MSEs) in state space models.

Within this setup, one of the most compelling approaches is the mixed-frequency VAR framework. However, computing the forecasts from this approach usually relies on serious dimensionality problems, especially when the number of time series or their frequency increases. Fortunately, the mixed-frequency VAR stated in state-space form can, alternatively, be estimated using Bayesian methods.

The parameter proliferation problem can also be addressed by means of the well known reduction dimensionality allowed by factor models. Since macroeconomic data are usually very collinear, it is reasonable to conjecture that they are multiple, indirect measurements of some low-dimensional underlying sources, which can be used to reproduce most of the variability of a data set, although typically they cannot be directly measured. Therefore, factor models have the additional appeal of computing indexes of the overall economic activity, which are very useful for tracking the economic developments.

Although much professional attention has recently been devoted to examining the pros and cons of these alternative forecasting approaches, it is difficult to rank them based purely on theoretical considerations. The matter is ultimately empirical, requiring detailed comparative assessment. However, while numerous empirical applications have been proposed in the literature, there is lack of comparative evaluation of the empirical performance of the different models. In this paper, we comprehensively examine the performance of the most significant models that have been suggested in the academic literature to compute short-term forecasts in economics for the same economy and time period.

The case we analyze is one of the most relevant for policy making, namely forecasting quarterly US GDP growth. The set of monthly indicators used to compute the forecasts comprises the monthly growth rates of industrial production, employment, income and sales, which become available in different time periods and with different publication 
delays. ${ }^{1}$ At any point $t$ in real time, we simply use the time- $t$ data vintage to extract the short-term forecast of the next unobserved US GDP figure. As time progresses, we re-estimate all the models for each period, always using the latest data vintage to compute the forecast. Therefore, the experiment mimics the day-to-day monitoring of the economic activity as it would have been developed in real time. Our results suggest that all the models that use indicators represent a massive improvement in forecasting over the pure autoregressive ones, with marginal differences across the different specifications. However, the nonlinear specification has the advantage of computing timely and accurate real-time inferences about the US business cycles.

Banbura, Giannone and Reichlin (2011) and Banbura, Giannone, Modugno and Reichlin (2013) provide complementary surveys of the short-term forecasting literature. The econometric framework used by Banbura, et al. (2011) is a large scale linear dynamic factor model, which is also one of the models used in the empirical application of Banbura et al. (2013). Although we consider linear factor models in this survey, they are treated as one alternative among a list of competing forecasting models. In addition, the models surveyed in this paper are applied to a small group of indicators for forecasting. The forecasting role of the number of time series in dynamic factor models has already been treated in Alvarez, Camacho and Perez Quiros (2012). Readers interested in forecasting with larger datasets are referred to the above mentioned surveys and the reviews of factor models developed in Bai and Ng (2008), Stock and Watson (2011) and Breitung and Choi $(2013) .^{2}$

The paper proceeds as follows. In Section 2, we introduce the notation and main characteristics of the data for short term forecasting. In section 3, we review the main models used for this purpose address the role of the number of series in factor models.

\footnotetext{
${ }^{1}$ Although the NBER Business Cycle Dating Committee does not have a fixed definition of economic activity, it acknowledges on its home page that these are the five indicators that examines to analyze the US business cycle conditions.

${ }^{2}$ After the paper was reviewed by the editor, we became aware of the working paper by Foroni and Marcellino (2013). In an independent research, they also survey some of the models that we consider. We differ from their work by focusing on different data features and by including an empirical application with a real time comparison of the reviewed models.
} 
In Section 4, we illustrate the forecasting performance of the different models reviewed in Section 3 through an empirical application. Finally, in Section 5, we conclude and propose some lines of further research.

\section{Short-term forecasting: the evil is in the details}

In this section, we examine a few common solutions to the short-term forecasting problem. After setting up the forecasting environment, we confront two forecasting strategies: direct and iterated multistep ahead forecasts. Next, we examine some solutions to the mixing frequency problem and to the presence of missing observations at the end of the sample, reflecting the unsynchronized data release dates.

\subsection{Forecasting environment and notation}

To compare across different modeling environments, it is very useful to establish a common notation. In the forecasting models that follow, we assume that the objective is to forecast a lower-frequency (quarterly) flow variable $\widetilde{y}_{\tau}^{q}$, whose logs are assumed to have a unit root so that the variable of interest is its quarterly growth rate, $y_{\tau}^{q}$. In this notation, the subscript refers to a quarterly time index $\tau=1,2, \ldots, \Upsilon_{y}$, and the superscript refers to quarterly growth rates. One significant example in macroeconomic analysis is the forecast of GDP growth rates.

This variable can be expressed at monthly frequency by setting, $y_{t}^{q}=y_{\tau}^{q}, \forall \tau=3 t,{ }^{3}$ where the subscript $t$ refers now to a monthly time index and $t=1,2, \ldots, T_{y}$. Hence, $y_{t}^{q}$ is observed only at months $t=3,6, \ldots, T_{y}$. Finally, it is convenient to define the underlying unobserved monthly growth rates $y_{t}^{m}$, where the subscript refers to a monthly time index $t=1,2, \ldots, T_{y}$ and the superscript refers to monthly growth rates.

We also assume that the information set used to forecast this variable typically include

\footnotetext{
${ }^{3}$ The quaterly variable $y_{t}^{q}$ at monthly frequency implies that, even though it is monthly, the variable refers to the three months growth rate over the previous three months. For example, refering to the GDP series, the variable $y_{t}^{q}$ for the month of November would refer to GDP growth in November, October and September over August, July and June. This is a number not reported every month by the statistical agencies but conceptually, it could be calculated.
} 
a set of higher-frequency flow indicators, which are collected in the $N$-dimensional vector $\widetilde{X}_{t}^{m}$, with $t=1,2, \ldots, T_{\widetilde{X}}$. If the logs of these indicators contain a unit root, they typically enter the forecasting equations in monthly growth rates, $X_{t}^{m}$, with $t=1,2, \ldots, T_{X}$. A typical example is a model that predicts GDP quarterly growth rates based on the monthly growth rates of some key economic indicators as industrial production, nonfarm payroll employment, personal income and retail sales.

For the sake of simplifying the example, let us start by assuming that there is only one monthly indicator, for example, industrial production, whose monthly growth rates are $x_{t}^{m}$, with $t=1,2, \ldots, T_{x}$. To estimate some of the forecasting models, it is convenient to define the skip sampled indicator $x_{t}^{3 m}$, which is constructed by picking every third observation of $x_{t}^{m}$ starting from the final observation $T_{x}$. More precisely, the skip sampled indicator is defined as $x_{t}^{3 m}=x_{t}^{m}, t=\ldots, T_{x}-6, T_{x}-3, T_{x}$.

Because the data are usually released in blocks and the releases follow a relatively stable calendar, the real-time forecasts are typically conditional on the same (updated) sets of data releases following somehow stylized schedules. An example of this forecast timeliness is depicted in Figure 1, which for simplicity only shows a stylized three-monthahead (one-quarter-ahead) forecast of the target variable $y_{\tau}^{q}$. Assume that at time $t^{*}$ we are interested in computing the forecast of $y_{T_{y}+3}^{q}\left(y_{\Upsilon_{y}+1}^{q}\right)$, which is observed until month $T_{y}$, from the information of the monthly indicator, which is available up to $T_{x}$. Typically, the monthly indicators are available sooner than the quarterly indicators, which imply that $T_{y}<T_{x}$. Accordingly, in this example the end-of-period forecasting problem consists is to compute an accurate forecast of $y_{T_{y}+3 \mid T_{x}}^{q}$.

\subsection{Direct versus iterated forecasts}

A commonplace example of multiperiod forecasting is the end-of-period forecast of the quarterly indicator $y_{\tau}^{q}$ from the information provided by the monthly indicator $x_{t}^{m}$. To isolate the problem of multiperiod forecasting from other problems of short-term forecasting, let us assume in this section that the interest lies in computing $h$-period-ahead forecasts of the quarterly indicator $y_{\tau}^{q}$ from the skip sampled indicator $x_{\tau}^{3 m}$, where both time series are assumed to be available up to the same quarter, i.e., $\Upsilon=\Upsilon_{y}=\Upsilon_{x}$. 
A forecaster making this multiperiod time series forecast faces a choice between using two forecasting strategies. The first strategy consists of computing direct $h$-step-ahead projections from the regression model

$$
y_{\tau+h}^{q}=\gamma_{0}+\gamma_{1} y_{\tau}^{q}+\gamma_{2} x_{\tau}^{3 m}+\epsilon_{\tau+h} .
$$

where $\epsilon_{\tau+h}$ is the disturbance term, which is not necessarily serially uncorrelated. In this case, the estimates of the parameters are the minimizers of the mean squared error of this $h$-step-ahead criterion function. Accordingly, the parameters $\gamma_{i}, i=0,1,2$, can be estimated by OLS in which the regressors are a constant, $y_{\tau}^{q}$ and $x_{\tau}^{3 m}$, and the dependent variable is $y_{\tau+h}^{q}$, by using observations from $\tau=1$ to $\tau=\Upsilon-h$. Using the estimated parameters $\widehat{\gamma}_{0}, \widehat{\gamma}_{1}$ and $\widehat{\gamma}_{2}$, the estimate of the optimal $h$-step-ahead projection $y_{\Upsilon+h \mid \Upsilon}^{q}$ can be obtained as $\widehat{\gamma}_{0}+\widehat{\gamma}_{1} y_{\Upsilon}^{q}+\widehat{\gamma}_{2} x_{\Upsilon}^{3 m}$.

The second forecasting strategy entails first estimating the autoregression

$$
y_{\tau}^{q}=\gamma_{0}+\gamma_{1} y_{\tau-1}^{q}+\gamma_{2} x_{\tau-1}^{3 m}+\epsilon_{\tau}
$$

where $\epsilon_{\tau}$ is a serially uncorrelated disturbance, with observations from $\tau=1$ to $\tau=\Upsilon$, then iterating upon that autoregression to obtain the multiperiod forecast. In the case of an iterated forecast, the estimate of the first one-period-ahead forecast $y_{\Upsilon+1 \mid \Upsilon}^{q}$, can be computed straightforwardly as $\widehat{\gamma}_{0}+\widehat{\gamma}_{1} y_{\Upsilon}^{q}+\widehat{\gamma}_{2} x_{\Upsilon}^{3 m}$. However, the second one-period-ahead forecast $y_{\Upsilon+1 \mid \Upsilon}^{q}$, cannot be directly computed as $\widehat{\gamma}_{0}+\widehat{\gamma}_{1} y_{\Upsilon+1 \mid \Upsilon}^{q}+\widehat{\gamma}_{2} x_{\Upsilon+1 \mid \Upsilon}^{3 m}$ since $x_{\Upsilon+1 \mid \Upsilon}^{3 m}$ is not available from the single-equation forecasting model.

This example illustrates a typical problem of short-term multivariate forecasting from single-equation models: each of the explanatory variables used in the estimating autoregression must be projected in separate time series models. Accordingly, the predictions of the variable of interest must be obtained in two steps. First, the explanatory indicators are forecast over the remaining forecasting horizons on the basis of auxiliary time series models. Second, these projections are used to roll the one-period model forward to obtain forecasts of the variable of interest.

In theory, Marcellino, Stock and Watson (2006) show that in linear specifications iterated forecasts are more efficient if the one-period ahead model is correctly specified, but 
direct forecasts are more robust to model misspecification. Iterative forecasts needs separate projections of the explanatory variables and the success in forecasting the variable of interest will rely on the accuracy of the auxiliary regressions. In addition, iterative forecasts entail estimating a large number of parameters, which could erode forecast performance.

However, which approach is better will ultimately be an empirical matter. Direct forecasts come from horizon-specific estimated models which may complicate the realtime forecasting procedure, especially when the forecasts are frequently updated. Using simulated out-of-sample methods with 170 U.S. monthly macroeconomic time series, Marcellino, Stock and Watson (2006) find that the iterated forecasts typically outperform direct forecasts, particularly, if the models can select long-lag specifications. These findings rely on linear specifications.

\subsection{Mixed frequencies}

Some of the major macroeconomic indicators, such as GDP, are available quarterly with a substantial delay. With the aim of obtaining early estimates of these low-frequency variables, analysts frequently focus on higher frequency economic indicators. The reason is that they, while showing high correlation with the low-frequency variables, contain more timely information.

The problem of mixed sampling frequencies is exemplified in Figure 2. This figure shows quarterly GDP and monthly industrial production growth rates for the period from the beginning of the 2008-2009 Great Recession to the latest available figures in February, 2011. According to the figure, the industrial production index appears to be an interesting procyclical indicator, in the sense that their monthly observations fluctuate between the quarterly GDP observations. However, GDP and industrial production are available at different frequencies.

Since the real-time forecasts will typically involve time series data sampled at different frequencies, any attempt to compute real-time forecasts from models that use several economic indicators must handle the problem of using mixed-frequency data. However, to compute the model estimates in traditional forecasting models, all the variables in the 
model must be sampled at the same frequency, which implies that the forecasters face one of the following two alternatives. Either the higher-frequency data must be aggregated to the lowest frequency or the lower-frequency data must be interpolated to the highest frequency.

In some empirical applications, the higher frequency is aggregated to the lower frequency by averaging, summing up, or by taking a representative corresponding value (for example, the third month of the quarter). These aggregations to the lower frequency are then plugged into a lower-frequency time series model. In some other applications, the lower frequency is interpolated to the higher frequency. Bridge equations and MIDAS models are significant examples.

Interpolation typically assumes that the lower-frequency variable is interpreted on some higher frequency which exhibits missing observations. Forecasting from interpolated time series is to project the series of interest, which contains missing observations, either on its own available data or on a set of series covering the whole sample. The methods usually employed in interpolating face the issues of how best to revise the data for the lower-frequency data to be compatible with the aggregated data and of how big the bias that emerges when the interpolated series are subsequently used in econometric analyses is. A significant contribution to this literature is Angelini, Henry, and Marcellino (2006).

Some of the most recent contributions to real-time forecasting from mixed frequency indicators are based on the method that assumes that the models operate at the highest frequency in the data. All the variables are assumed to be generated, but not necessarily observed, at the highest frequency. Hence, the variables observed at a lower frequency are viewed as being periodically missing and the system can be conveniently represented as a state-space model. Using the Kalman filter with missing data, one can reconstruct the lower-frequency series as if they were observed at the higher frequencies. Extensions of VAR and dynamic factor models that account for mixed frequencies are two interesting approaches. 


\subsection{Unbalanced data sets}

In addition to the frequency sampling problem, the (timely) day-to-day monitoring of the economic activity has to deal with the typical lack of synchronicity that characterizes the daily flow of macroeconomic information. For example, hard indicators, which are based on economic activity data, usually exhibit longer publication delays than soft indicators, which are based on surveys. With a few exceptions, financial indicators are available with no significant delays.

As we mentioned in the introduction, not accounting for this publication pattern would imply that the users of traditional forecasting models face severe costs. Typically, the forecaster has to wait until having a balanced panel, losing the valuable information contained in the promptly issued indicators at the time of the assessments. Therefore, the inferences actually refer to the past. In this context, the lack of a timely comprehensive economic picture implies that policy reactions may occur some months after a significant slowdown or an acceleration in the economy. As we show below, this problem is especially important around business cycle peaks or troughs, where there may be only weak evidence from some promptly published economic indicators that the economy is changing the business cycle phase. In this paper, we only consider models that deal with asynchronously published economic indicators, which allow the models to compute real-time forecasts on the basis of timely updated data.

\subsection{Assessment of the forecasting performance}

The economic forecasts are intended to be used in many aspects of economic life: business planing, health care decisions, national and local budgeting, financial management, and monetary and fiscal policy. Therefore, a responsible use of the outcome of a forecasting model must be accompanied by an exhaustive analysis of the actual empirical performance that the model would exhibit in practice.

Among many others, Stark and Croushore (2002) suggest that the analysis of in-sample forecasting performance of competitive models is questionable since the results can be deceptively less conclusive when using real-time vintages. Basically, this happens because the in-sample analysis misses three aspects of real-time forecasting: (i) the recursive es- 
timation of the model parameters; (ii) the real-time data flow, i.e. the fact that data are released at different point in time; and (iii) the real-time data revisions. Accordingly, Koenig, Dolmas and Piger (2003) suggest the use of real-time data for estimation and forecast evaluation purposes whenever possible. Recent examples of forecasting from real-time data vintages are Giannone, Reichlin and Small (2008) and Clements and Galvao (2009) for US data, and Diron (2008) and Camacho and Perez Quiros (2010) for Euro area data.

However, although developing real-time data sets is conceptually simple, producing real-time vintages is sometimes unfeasible since the historical records of many time series are not available. One alternative, employed for example by Stock and Watson (2002), is to develop an out-of-sample analysis, in which (i) holds. The method consists of computing forecasts from successive enlargements of a partition of the latest available data set. It begins with data from the beginning of the sample until one predefined period. Using this sample the model is estimated and the $h$-period-ahead forecasts are computed. Then, the sample is updated by one period, the model is reestimated and the forecasts are computed again. The forecasting procedure continues iteratively until the final forecast, which is computed from a model that uses the complete latest available data vintage.

In the context of forecasting from multivariate models, an interesting alternative to the out-of-sample forecasting analysis is the pseudo real-time forecasting exercise. Although the latter method is also based on successive enlargements of the latest available data set, it differs from the out-of-sample analysis since, when constructing the data vintages, the analyst takes into account the real time data flow (and hence the publication lags). The proposal is based on trying to mimic as closely as possible the real-time analysis that would have been performed by a potential user of the models when forecasting, at each period of time, on the basis of the different vintages of data sets. Thus, the experiment considers that the releases of each vintage contain missing data at the end of the sample reflecting the calendar of data releases. Accordingly, only (i) and (ii) hold in these experiments, which are labeled "pseudo" because the vintages are not obtained in pure real time but from different partitions of the latest available data. Therefore, this strategy does not account for data revisions.

The extent to which using data vintages constructed from the latest-available data 
could potentially give a misleading picture of the actual forecasting accuracy of the model is ultimately empirical. The literature show mixed results in this context. Diron (2008) and Giannone, Reichlin and Small (2008) find that the average reliability measures of pseudo real-time exercises seem valid, since the forecasts tend to be similar regardless of whether they are based on preliminary or revised data. By contrast, from the earliest paper by Denton and Kuiper (1965) to the recent analyses of Molodtsova, Nikolsko-Rzhevskyy, and Papell (2008) and Clements and Galvao (2009) the literature is full of examples that find significant differences in the forecasts depending on whether real-time data or latestavailable data are used. A good survey in forecasting comparison methods can be found in West (2006).

\section{Models}

There is a growing body of the literature dealing with the problems of short-term forecasting reviewed above. Although the list of surveyed models tries to offer a comprehensive overview of the recent advances in the literature, it does not pretend to be exhaustive. To facilitate the exposition, all the models are described in the simplest case of forecasting the variable of interest by using a single indicator. However, they can be extended easily to include multiple indicators, as we do in the empirical application.

\subsection{Bridge equations}

Bridge equations have been used extensively to compute forecasts from mixed frequency data, as in Ingentino and Trehan (1996), Runstler and Sedillot (2003), Baffigi, Golinelli, Parigi (2004) and Diron (2008). ${ }^{4}$ They are essentially single-equations time series models based on regressions relating high-frequency indicators which are aggregated to lower frequencies and plugged into the low-frequency time series model. The aggregations can be obtained by averaging, summing up, or by taking a representative corresponding value, as in the skip sampled indicator.

As single-equation specifications, when the forecasts are computed iteratively, the high-

\footnotetext{
${ }^{4}$ Further references on bridge equations can be found in these papers.
} 
frequency data must be forecast up to the desired forecast horizon in separate time series models. To overcome this additional complexity, in this survey we focus on direct forecasts. ${ }^{5}$ To facilitate the analysis, let $x_{\tau}^{3 m}$ be the skip sampled indicator and assume that $\Upsilon_{y}=\Upsilon_{x}=\Upsilon$. The bridge equation $h / 3$-quarter-ahead forecasting model, $h=3,6, \ldots$, can be stated as

$$
y_{\tau}^{q}=\alpha_{0}+c^{q}\left(L_{q}, K_{1}\right) x_{\tau-h / 3}^{3 m}+\epsilon_{\tau},
$$

where $\varepsilon_{\tau}$ is a serially uncorrelated disturbance, $c^{q}\left(L_{q}, K_{1}\right)$ is the quarterly lag polynomial $c_{0}^{q}+c_{1}^{q} L_{q}^{1}+\ldots+c_{K_{1}}^{q} L_{q}^{K_{1}}$ and $L_{q}$ is the quarterly backshift operator. In this case, the $h / 3$-quarter-ahead forecasts can be computed by estimating the model with quarterly data of $y_{\tau}^{q}$ up to $\tau=\Upsilon$ and quarterly data of the skip sampled indicator $x_{\tau}^{3 m}$ up to $\tau=\Upsilon-h / 3$. Then, the estimated parameters can be used to compute the projections of $x_{\Upsilon-K_{1}}^{3 m}, x_{\Upsilon-K_{1}+1}^{3 m}, \ldots, x_{\Upsilon}^{3 m} \cdot{ }^{6}$

Standard bridge equation models can be extended in two ways. First, the process used to transform left-hand and right-hand variables into the same frequency usually discards a lot of potentially useful information and makes the detection of the actual relation between the variables difficult. Second, the model cannot capture the latest available information provided by the higher-frequency indicators, which is ignored in this proposal whenever $T_{y}<T_{x}$, since it requires knowing the indicators for the entire quarter. Otherwise, the monthly data must be forecast in separate time series models.

Inspired in the MIDAS specification, a bridge equation $h$-month-ahead prediction model that takes into account the limitations described above, can be stated as follows:

$$
y_{\tau}^{q}=\alpha_{0}+c(L, K) x_{t-h}^{3 m}+\epsilon_{\tau},
$$

where

$$
c(L, K)=c_{0}+c_{1} L^{1}+\ldots+c_{K} L^{K}
$$

is the monthly lag polynomial, $L$ is the monthly backshift operator, and usually $K>>K_{1}$. Note that, for convenience, the right-hand-side time indicator is expressed in monthly

\footnotetext{
${ }^{5}$ To save space, linkage models are omitted from this survey. In these models, the forecasts are generated at different frequencies and are combined to improve the forecasting accuracy of the lower-frequency time series.

${ }^{6}$ To exploit information from several monthly predictors, bridge equations are sometimes pooled.
} 
terms since the higher frequency indicator is not aggregated to obtain quarterly observations before entering the model. In addition, the $h$-month-ahead forecasts can be computed in real time by incorporating the flow of monthly data as they become available. The model is estimated with quarterly data of $y_{\tau}^{q}$ up to $\tau=\Upsilon_{y}$ (or $T_{y}$ on a monthly basis), and the weighted sum of monthly observations $x_{T_{x}-K-h}, x_{T_{x}-K-h+1}, \ldots, x_{T_{x}-h}$. The corresponding estimated parameters are then used to compute the projections of $x_{T_{x}-K}$, $x_{T_{x}-K+1}, \ldots, x_{T_{x}}$.

Note that, in this framework the polynomial lag structures are left unconstrained. Therefore, there is a potentially large number of lags of the monthly variables that are likely to be significant, which implies that the number of parameters to be estimated by the models would sometimes become unfeasible, especially when they refer to very high frequency (weekly or daily) data. A lag polynomial a la Almon, could be a solution.

\subsection{MIxed DAta Sampling (MIDAS) regression}

Bridge equation models are parsimonious but usually require the user to estimate a potentially large number of parameters. To solve the problem of parameter proliferation while preserving some timing information Ghysels, Santa-Clara, and Valkanov (2004) propose the MIDAS model. Given a weighting function $b(L, K, \theta)$, the MIDAS model can be adapted to our $h$-month-ahead forecasting framework as follows

$$
y_{\tau}^{q}=\alpha_{0}+\alpha_{1} b(L, K, \theta) x_{t-h}^{3 m}+\varepsilon_{\tau}
$$

where $\varepsilon_{\tau}$ is a serially uncorrelated disturbance. Accordingly, the forecasts are computed by estimating the model with quarterly data of $y_{\tau}^{q}$ up to $\tau=\Upsilon_{y}$ (or $T_{y}$ on a monthly basis) and quarterly data of the skip sampled indicator $x_{t}^{3 m}$ up to $t=T_{x}-h$. Then, the model uses the estimated parameters to compute the projections of $x_{T_{x}-K-h}, x_{T_{x}-K-h+1}$, $\ldots, x_{T_{x}-h}$.

To overcome the parameter proliferation problem of bridge equations, the MIDAS framework constrains the polynomial lag structures with nonlinear functional specifications, the weighting functions $b(L, K, \theta)$. They can have any number of functional forms whenever they achieve flexibility, impose reasonable constraints on the parameter dynam- 
ics and maintain the desired parsimony. In practice, by playing with different weighting functions, one might impose different prior beliefs on the weights that should be given to lagged values of the explanatory variables. For example, the weights can be estimated without restriction, they can preserve the within-quarter effects but have different effects across months, or they can be restricted to the case in which the explanatory variables are aggregated to the lower frequency by averaging, summing up, or by taking a representative corresponding value, as in traditional bridge equation models.

Ghysels, Sinko, and Valkanov (2007) examines various specifications of MIDAS regression polynomials as finite polynomials, infinite polynomials, autoregressive augmentations, rational polynomials and step functions. In this paper, we focus on the finite lag polynomial

$$
b(L, K, \theta)=b_{0}(\theta) L^{0}+b_{1}(\theta) L^{1}+\ldots+b_{K}(\theta) L^{K},
$$

where the weights $b_{k}(\theta), \theta=\left(\theta_{1}, \theta_{2}\right)$ are based on the exponential Almon weighting function

$$
b_{k}(\theta)=\frac{\exp \left(\theta_{1} k+\theta_{2} k^{2}\right)}{\sum_{k=0}^{K} \exp \left(\theta_{1} k+\theta_{2} k^{2}\right)} .
$$

According to this parameterization, the lag coefficients are always positive and sum up to unity. One of the main advantages of this functional form is that it is quite flexible and allows for various shapes with only two parameters. To illustrate the characteristics of the exponential Almon lag, Figure 3 plots various parameterizations of this polynomial weighting function. For $\theta_{1}=\theta_{2}=0$, the polynomial imposes equal weights. For some combinations of the parameters, the weights decline, which refer to the case in which more weight is given to the months of the explanatory variable that are more contemporaneous to the observed $x_{t}^{3 m}$. For other combinations, weights can also produce hump shapes. ${ }^{7}$

While the exponential Almon weighting function is a practical parsimonious way of allowing lags of explanatory variables, it may be difficult to obtain accurate estimates of its parameters from numerical nonlinear optimization procedures. Figure 3 illustrates the difficulty in estimating parameters of the weighting function in practice since for very

\footnotetext{
${ }^{7}$ The rate of decline will determine how many lags are included in MIDAS regression, $K$. The lag length can be either data driven or large enough to ensure that the declining weighting function falls to zero.
} 
small changes in the polynomial parameters produce large effects in the evolution of the weights. For $\theta_{1}=0.001$, the decays are very smooth for $\theta_{2}=-0.001$ and the weights remain steadily about 0.04 for any lag of the explanatory variable. However, the decays are much sharper when is $\theta_{2}=-0.009$. The impacts of current values of the explanatory variable start at about 0.1 but they become negligible for more than 15 months lagged values. $^{8}$

The natural extension of this simple MIDAS model is to include lags of the dependent variable as regressors. Although conceptually it seems that the MIDAS model can be easily adapted to include autoregressive components, Ghysels et al. (2007) pointed out that this is not straightforward. If an autoregressive component is added to the MIDAS specification unrestricted, it would generate a seasonal response of the variable of interest to $x_{t}^{3 m}$ irrespective of whether or not $x_{t}^{3 m}$ displays a seasonal pattern. Fortunately, Clements and Galvao (2008) propose an easy solution which is based on including the autoregressive lags as a common factors and ensures a smooth impulse response function. Consider simply adding one lower-frequency lag for $h$-step-ahead forecasting

$$
y_{\tau}^{q}=\alpha_{0}+\lambda y_{\tau-1}^{q}+\alpha_{1} b(\theta, K, L)(1-\lambda L) x_{t-h}^{3 m}+\varepsilon_{\tau+h},
$$

which is frequently referred to as autoregressive MIDAS model.

In contrast to bridge equation models, in the MIDAS framework the polynomial lag structures are constrained via nonlinear functional specifications, which implies that it requires nonlinear estimation procedures. The referenced literature establishes that estimating methods based on nonlinear least squares are consistent estimators for the MIDAS models. In addition, estimating the nonlinearities imposed by the restricted lag polynomial could be troublesome, so using a large variety of initial parameter specifications to check for the robustness of the results is clearly advisable. To facilitate the convergence in optimization procedures, the empirical applications usually impose constraints in the estimation of the Almon weighting function.

\footnotetext{
${ }^{8}$ Alternatively, for fixed $\theta_{1}$ and large negative values of $\theta_{2}$, the weighting function does not change significantly for large different valued of $\theta_{2}$. In these cases, regardless the value of $\theta_{2}$, the weight is 1 for $k=0$, and 0 otherwise.
} 
It is worth noting that the MIDAS forecast is $h$-dependent, and thus has to be reestimated for multistep forecasts according to the information available at the time of the forecast. For each $h$, the observed variables must be re-organized according to the forecasting horizon, which makes it necessary to manage $h$ different forecasting models at each forecast time. In addition, since the model changes from month to month, it is necessary to re-estimate the models when new observations for the indicator are released.

Since MIDAS models were introduced less than ten years ago, they are a prolific source of recent extensions. First, Marcellino and Schumacher (2010) merge factor and MIDAS approaches in the Factor-MIDAS model, so augmenting the MIDAS regressions with the factors extracted from a large dataset in high frequency. Although some theoretical work is required, we think that this is an interesting area to develop further research. ${ }^{9}$ Second, Ghysel et al. (2007) show that MIDAS models can be extended to account for nonlinearities. Galvao (2013) with smooth transitions and Guerin and Marcellino (2013) with Markov-swicthing dynamics are two illustrative examples. Third, Bai, Ghysels, and Wright (2012) interpret the MIDAS regression as a reduced form representation of the linear projection that emerges from a state space model approach. However, they show that the Kalman filter is more prone to specification errors and usually falls into the curse of dimensionality since it requires the specification of a full state space system of all the equations. Finally, Ghysels (2012), extends the MIDAS regression to a multivariate framework.

For interested readers, several recent surveys on the topic of MIDAS models are Andreou, Ghysels, and Kourtellos (2011), who review extensively the MIDAS models, Armesto, Engemann, and Owyang (2010), who provide a very intuitive introduction to MIDAS regressions and Ghysels and Valkanov (2012), who discuss volatility models and mixed data sampling.

\footnotetext{
${ }^{9}$ For example, it would be interesting to check if computing the forecasts in two steps (extracting the factors and using then in MIDAS) is preferable to mixing the frequencies in the factor model directly.
} 


\subsection{Mixed Frequency Vector Autoregression (MF-VAR)}

To exploit the advantages of using economic indicators that are available at different frequencies, one alternative to single-equation models is the mixed-frequency Vector Autoregression (MF-VAR) model. Since the early proposal by Zadrozny (1990), the approach of the method is to assume that the model operates at the highest frequency in the data. Hence, all variables are assumed to be generated, but not necessarily observed, at this highest frequency, and thus can be used to produce forecasts of any variable at this frequency. Variables which are observed at a lower frequency are viewed as being periodically missing and can be conveniently represented through a state-space model, in which the state-transition equation is given by a VAR at the higher frequency and the measurement equation relates the observed series to the underlying, potentially unobserved, lower frequency variables that are stacked in the state vector. ${ }^{10}$

Let us assume that the level of quarterly $\widetilde{y}_{\tau}^{q}$ can be decomposed as the sum of three unobservable monthly values that correspond to quarter $\tau, \widetilde{y}_{t}^{q}, \widetilde{y}_{t-1}^{q}, \widetilde{y}_{t-2}^{q}$. For instance, the GDP for the third quarter of a given year is the sum of the GDP corresponding to the three months of the third quarter. Among others, Mariano and Murasawa (2003) have shown that if the sample mean of the three within quarter monthly observations can be well approximated by the geometric mean, then the quarterly growth rates can be decomposed as weighted averages of monthly growth rates. In particular, the quarterly growth rate, $y_{\tau}^{q}$, is approximated by the weighted sum of five monthly growth rates:

$$
y_{\tau}^{q}=\frac{2}{3} y_{t}^{m}+\frac{1}{3} y_{t-1}^{m}+y_{t-2}^{m}+\frac{1}{3} y_{t-3}^{m}+\frac{2}{3} y_{t-4}^{m}
$$

It is worth mentioning that in a related paper, Aruoba, Diebold and Scotti (2009) avoid the geometric approximation but at the cost of assuming that the trend of the time series can be well described by deterministic trends. However, these authors have recently acknowledged that the benefits of moving to the geometric approximation of flow data exceeded the costs of assuming deterministic trends and, in the current versions of their index of business cycle conditions, which is updated at the Federal Reserve Bank of

\footnotetext{
${ }^{10}$ One noticeable recent exception is Ghysels (2012), whose approach does not rely on latent processes/shocks representations, in part inspired in MIDAS regressions.
} 
Philadelphia, they use the geometric approximation as well.

The latent month-on-month growths of GDP and the monthly indicator, $y_{t}^{m}$ and $x_{t}^{m}$, are then assumed to follow a bivariate $\operatorname{VAR}(p)$ process. Without loss of generality, to facilitate the description of the model we assume that $p=1$ and that there is only one monthly indicator. ${ }^{11}$ In this case, the model is

$$
\left(\begin{array}{c}
y_{t}^{m} \\
x_{t}^{m}
\end{array}\right)=\left(\begin{array}{l}
c_{1} \\
c_{2}
\end{array}\right)+\left(\begin{array}{ll}
c_{11} & c_{12} \\
c_{21} & c_{22}
\end{array}\right)\left(\begin{array}{c}
y_{t-1}^{m} \\
x_{t-1}^{m}
\end{array}\right)+\left(\begin{array}{l}
v_{1 t} \\
v_{2 t}
\end{array}\right),
$$

where $\left(v_{1 t}, v_{2 t}\right)^{\prime} \sim i i N(0, \Omega)$, with $E\left(v_{1 t}^{2}\right)=\omega_{1}^{2}, E\left(v_{2 t}^{2}\right)=\omega_{2}^{2}$, and $E\left(v_{1 t} v_{2 t}\right)=\omega_{12}$.

For clarity, let us start by assuming that all variables are always observed at a monthly frequency. The MF-VAR specification can be represented as state-space models. The measurement equation, $Y_{t}=H \beta_{t}+E_{t}$, with $E_{t} \sim$ iid $(0, R)$, can be defined as

$$
\left(\begin{array}{c}
y_{t}^{q} \\
x_{t}^{m}
\end{array}\right)=\left(\begin{array}{cccccc}
\frac{2}{3} & \frac{1}{3} & 1 & \frac{1}{3} & \frac{2}{3} & 0 \\
0 & 0 & 0 & 0 & 0 & 1
\end{array}\right)\left(\begin{array}{c}
y_{t}^{m} \\
y_{t-1}^{m} \\
y_{t-2}^{m} \\
y_{t-3}^{m} \\
y_{t-4}^{m} \\
x_{t}^{m}
\end{array}\right)
$$

where $E_{t}=0_{2 \times 1}$ and $R=0_{2 \times 2}$. The transition equation, $\beta_{t}=c+F \beta_{t-1}+V_{t}$, with $V_{t} \sim \operatorname{iid}(0, Q)$, can be stated as

$$
\left(\begin{array}{l}
y_{t}^{m} \\
y_{t-1}^{m} \\
y_{t-2}^{m} \\
y_{t-3}^{m} \\
y_{t-4}^{m} \\
x_{t}^{m}
\end{array}\right)=\left(\begin{array}{c}
c_{1} \\
0 \\
0 \\
0 \\
0 \\
c_{2}
\end{array}\right)+\left(\begin{array}{cccccc}
c_{11} & 0 & 0 & 0 & 0 & c_{12} \\
1 & 0 & 0 & 0 & 0 & 0 \\
0 & 1 & 0 & 0 & 0 & 0 \\
0 & 0 & 1 & 0 & 0 & 0 \\
0 & 0 & 0 & 1 & 0 & 0 \\
c_{21} & 0 & 0 & 0 & 0 & c_{22}
\end{array}\right)\left(\begin{array}{c}
y_{t-1}^{m} \\
y_{t-2}^{m} \\
y_{t-3}^{m} \\
y_{t-4}^{m} \\
y_{t-5}^{m} \\
x_{t-1}^{m}
\end{array}\right)+\left(\begin{array}{c}
v_{1 t} \\
0 \\
0 \\
0 \\
0 \\
v_{2 t}
\end{array}\right)
$$

\footnotetext{
${ }^{11}$ Models with higher lag orders and additional monthly indicators can be derived in a straightforward manner by modifying the VAR specification, the state vector and the system matrices accordingly.
} 
where

$$
Q=\left(\begin{array}{cccccc}
\omega_{1}^{2} & 0 & 0 & 0 & 0 & \omega_{12} \\
1 & 0 & 0 & 0 & 0 & 0 \\
0 & 1 & 0 & 0 & 0 & 0 \\
0 & 0 & 1 & 0 & 0 & 0 \\
0 & 0 & 0 & 1 & 0 & 0 \\
\omega_{12} & 0 & 0 & 0 & 0 & \omega_{2}^{2}
\end{array}\right)
$$

$H, R, c, F$ and $Q$ are the so called system matrices. The Kalman filter could be used to infer unobserved components.

In empirical applications, $y_{t}^{q}$ is not observed two months of each quarter and at the end of the sample due to the high publication lag that characterizes the GDP data flow. However, the unobserved cells can be treated as missing observations and maximum likelihood estimation of a linear Gaussian state-space model with missing observations can be applied straightforwardly after a subtle transformation of the system matrices.

As described in Mariano and Murasawa (2010), the missing observations can be replaced with random draws $\vartheta_{t}$, whose distribution cannot depend on the parameter space that characterizes the Kalman filter. Thus, the likelihood function of the observed data and that of the data whose missings are replaced by the random draws are equivalent up to scale. In particular, we assume that the random draws come from $N\left(0, \sigma_{\vartheta}^{2}\right)$. In addition, the measurement equation must be transformed conveniently in order to allow the Kalman filter to skip the missing observations when updating.

Let $Y_{i t}$ be the $i$-th element of the vector $Y_{t}$ and $R_{i i}$ its variance. Let $H_{i}$ be the $i$-th row of the matrix $H$ which has $\varsigma$ columns and let $0_{1 \varsigma}$ be a row vector of $\varsigma$ zeroes. The 
measurement equation can be replaced by the following expressions

$$
\begin{aligned}
Y_{i t}^{*} & =\left\{\begin{array}{cc}
Y_{i t} & \text { if } Y_{i t} \text { observable } \\
\vartheta_{t} & \text { otherwise }
\end{array},\right. \\
H_{i t}^{*} & =\left\{\begin{array}{cc}
H_{i} & \text { if } Y_{i t} \text { observable } \\
0_{1 \varsigma} & \text { otherwise }
\end{array},\right. \\
E_{i t}^{*} & =\left\{\begin{array}{cc}
0 & \text { if } Y_{i t} \text { observable } \\
\vartheta_{t} & \text { otherwise }
\end{array}\right. \\
R_{i i t}^{*} & =\left\{\begin{array}{cc}
0 & \text { if } Y_{i t} \text { observable } \\
\sigma_{\vartheta}^{2} & \text { otherwise }
\end{array}\right.
\end{aligned}
$$

This trick leads to a time-varying state space model with no missing observations so the Kalman filter can be directly applied to $Y_{t}^{*}, H_{t}^{*}, E_{t}^{*}$, and $R_{t}^{*}$.

The way missing observations are treated implies that the filter, through its implicit signal extraction process, puts no weight on missing observations in the computation of the factors. In this way, when no observation is available, the filter produces a forecast of the common factors.

The Kalman filter is extremely useful for computing real-time forecasts that need to be updated frequently. This is easy to see when the treatment of missing values is adopted for the computation of these forecasts since one can regard the future values of the time series as a set of missing observations. After the last observation, we add a series of missing values to the data set and carry on with the Kalman filter that accounts for missing data. Then, the model treats the future observations as missing values in the way described above.

In addition, one can use the basic filtering and smoothing algorithms for Markovswitching state-space models and the maximum likelihood estimation of the unknown parameters of the models proposed by Filardo (1994) to extend the linear MF-VAR to nonlinear contexts. A significant example is Camacho (2013), who extend the linear MF-VAR proposed by Mariano and Murasawa (2010) to account for Markov-switching dynamics.

The forecasts based on Kalman filters usually rely on serious dimensionality problems, 
especially when the number of time series or their frequency increase. For example, a MF-VAR model to forecast GDP from four monthly indicators and $p=2$ requires estimating 70 parameters. Several approaches have recently emerged to cope with the high dimensionality of the parameter space. First, if the cross-autocorrelations in the system are small, one approach is to take all the off-diagonal elements of the polynomial matrices as zero. However, such constraints may not always be appropriate and will be dictated by the application at hand. Second, in the context of MF-VAR models, they can be cast in state-space forms and the parameters can be estimated using Bayesian methods. Eraker, Chiu, Foerster, Kim, and Seoane (2011) or Schorfheide and Song (2011) are significant examples.

\subsection{Linear factor models}

The parameter proliferation problem can also be addressed by means of the well known reduction dimensionality allowed by factor models. Since macroeconomic data are usually very collinear, it is reasonable to conjecture that they are multiple, indirect measurements of some low-dimensional underlying sources, which can be used to reproduce most of the variability of a data set.

\subsubsection{Small scale factor models}

Let us assume that a handful set of indicators used in the model are somehow related to the overall economic conditions. Based on the seminal proposal by Stock and Watson (1991) to construct a coincident indicator of the economic activity, we consider a single-index dynamic factor model such that each variable can be written as the sum of two mutually uncorrelated stochastic components. ${ }^{12}$ The first component, $f_{t}$, is assumed to be common for all the time series in the model and typically represents the overall business cycle in macroeconomic applications. The second component, $u_{i t}$, refers to the idiosyncratic dynamics of each indicator.

Accordingly, the latent month-on-month growths of GDP and the monthly indicator,

\footnotetext{
${ }^{12}$ Allowing for more than one factor is straightforward.
} 
$y_{t}^{m}$ and $x_{i t}^{m}$, are then assumed to admit the following factor decomposition

$$
y_{t}^{m}=\beta_{0} f_{t}+u_{0 t},
$$

and

$$
x_{i t}^{m}=\beta_{i} f_{t}+u_{i t},
$$

for $i=1, \ldots, N$, where $N$ is the number of monthly indicators used in the analysis.

To complete the statistical specification of the model, both components are modelled by using the standard techniques of linear autoregressive time series. Therefore, the dynamics of the model is achieved by assuming that the factor and the idiosyncratic components evolve according to autoregressive processes:

$$
\begin{aligned}
f_{t} & =\phi_{f 1} f_{t-1}+\ldots+\phi_{f p_{f}} f_{t-p_{f}}+\epsilon_{f t}, \\
u_{j t} & =\phi_{j 1} u_{1 t-1}+\ldots+\phi_{j p_{j}} u_{j t-p_{j}}+\epsilon_{j t},
\end{aligned}
$$

where $\epsilon_{f t} \sim i i N\left(0, \sigma_{f}^{2}\right), \epsilon_{j t} \sim i i N\left(0, \sigma_{j}^{2}\right)$, with $j=0,1, \ldots, N$. All the covariances are assumed to be zero. To achieve identification, the variance of the noise associated to the common factor, $\sigma_{f}^{2}$, is assumed to be one. ${ }^{13}$

This model can be easily cast in steady state representation. To illustrate this, let us assume that $p_{f}=p_{j}=1$ and that $N=1$. In this case, the matrices of the measurement equation are:

$$
\begin{aligned}
Y_{t} & =\left(y_{t}^{q}, x_{t}^{m}\right)^{\prime}, \\
H & =\left(\begin{array}{ccccccccccc}
\frac{\beta_{0}}{3} & \frac{2 \beta_{0}}{3} & \beta_{0} & \frac{2 \beta_{0}}{3} & \frac{\beta_{0}}{3} & \frac{1}{3} & \frac{2}{3} & 1 & \frac{2}{3} & \frac{1}{3} & 0 \\
\beta_{1} & 0 & 0 & 0 & 0 & 0 & 0 & 0 & 0 & 0 & 1
\end{array}\right), \\
\beta_{t} & =\left(f_{t}, f_{t-1}, f_{t-2}, f_{t-3}, f_{t-4}, u_{0 t}, u_{0 t-1}, u_{0 t-2}, u_{0 t-3}, u_{0 t-4}, u_{1 t}\right)^{\prime}, \\
E_{t} & =0_{2 \times 1}, \\
R & =0_{2 \times 2} .
\end{aligned}
$$

\footnotetext{
${ }^{13}$ This identifying assumption is standard in dynamic factor models.
} 
The matrices of the transition equation are:

$$
\begin{aligned}
c & =0_{11 \times 1}, \\
F & =\left(\begin{array}{ccccccccccc}
\phi_{f 1} & 0 & 0 & 0 & 0 & 0 & 0 & 0 & 0 & 0 & 0 \\
1 & 0 & 0 & 0 & 0 & 0 & 0 & 0 & 0 & 0 & 0 \\
0 & 1 & 0 & 0 & 0 & 0 & 0 & 0 & 0 & 0 & 0 \\
0 & 0 & 1 & 0 & 0 & 0 & 0 & 0 & 0 & 0 & 0 \\
0 & 0 & 0 & 1 & 0 & 0 & 0 & 0 & 0 & 0 & 0 \\
0 & 0 & 0 & 0 & \phi_{01} & 0 & 0 & 0 & 0 & 0 & 0 \\
0 & 0 & 0 & 0 & 1 & 0 & 0 & 0 & 0 & 0 & 0 \\
0 & 0 & 0 & 0 & 0 & 1 & 0 & 0 & 0 & 0 & 0 \\
0 & 0 & 0 & 0 & 0 & 0 & 1 & 0 & 0 & 0 & 0 \\
0 & 0 & 0 & 0 & 0 & 0 & 0 & 1 & 0 & 0 & 0 \\
0 & 0 & 0 & 0 & 0 & 0 & 0 & 0 & 0 & 0 & \phi_{11}
\end{array}\right), \\
V & =\left(\epsilon_{f t}, 0,0,0,0, \epsilon_{0 t}, 0,0,0,0, \epsilon_{1 t}\right), \\
Q & \operatorname{diag}\left(\sigma_{f}^{2}, 0,0,0,0, \sigma_{0}^{2}, 0,0,0,0, \sigma_{1}^{2}\right) .
\end{aligned}
$$

So far, our discussion has assumed that all the variables used in $Y_{t}$ are observed. However, the variables entering the models exhibit missing data either because they are available only at some periods (in the case of quarterly observations), because they are not available at the end of the sample (in the case of variables with larger publication delays), or because they refer to the future (in the case of forecasting). This missing data problem appears in Mariano and Murasawa (2003), who extend the Stock-Watson approach to construct a coincident index of business cycles that uses both monthly and quarterly indicators. These authors were among the first to handle the missing observations by dropping the corresponding observation equations in each recursion of the Kalman filter in the way described below for MF-VAR models.

Several empirical applications of these models have appeared in the literature. Using Portuguese data, Nunes (2005) illustrates the usefulness of the model to compute a monthly index of coincident indicators as well as estimates of quarterly real GDP growth. Aruoba, Diebold and Scotti (2009) incorporate indicators measured at high frequencies to 
develop the very popular business conditions index of the US economy, which is updated by the Philadelphia Fed as new observations arrive. Camacho and Perez Quiros (2010) set out the Euro-STING dynamic factor model to compute short-term forecasts of the euro area GDP growth in real-time, which is able to forecast the euro area GDP growth as well as professional forecasters. Aruoba and Diebold (2010) focus not only on real macroeconomic activity indexes but also on an inflation index and its interaction with the real activity index. With Spanish data, Camacho and Domenech (2012) extend the approach of Camacho and Perez Quiros (2011) to include leading financial indicators. Finally, dal Bianco, Camacho and Perez Quiros (2012) propose a fundamentals-based dynamic factor model to forecast the weekly changes in the euro-dollar rate.

\subsubsection{The role of $N$ in factor models}

Due to the recent advances in information technology, there are thousands of time series available in real time whose information could be used to forecast the variable of interest. Significant examples are disaggregations of time series indicators, which can be obtained with an unprecedented degree of disaggregation by sectors, kind of goods, regions and so on; and financial variables, which can be obtained at daily, hourly or even higher frequencies. Aruoba, Diebold and Scotti (2009) have recently assessed that comparative assessment of experiences and results from forecasting with a relatively small set of preselected indicators versus forecasting from relatively large set of indicators would be a good place to develop further analyses.

To address the role of using many predictors in short-term forecasting, we propose the following framework. To facilitate the analysis, the goal is to compute one-period forecasts of $y_{t}$ from $N$ candidate predictors $x_{t}^{(N)}=\left(x_{1 t}, \ldots, x_{N t}\right)^{\prime}$, with $t=1, \ldots, T$, where $T$ is large $N$ could be large. The forecasting equation is

$$
y_{t+1}=\alpha_{0}+\sum_{j=1}^{p} \alpha_{j} y_{t-j}+\gamma^{\prime} x_{t}^{(N)}+e_{t+1},
$$

where $\gamma=\left(\gamma_{1}, \ldots, \gamma_{N}\right)^{\prime}$ and the one-period forecast error, $e_{t+1}$, is white noise. When the number of predictors becomes large, the estimation of the model is not be feasible. In 
these cases, it is standard to assume that the predictors admit a factor representation

$$
x_{t}^{(N)}=B F_{t}+u_{t}
$$

where $F_{t}=\left(f_{1 t}, \ldots, f_{r t}\right)^{\prime}$ is the vector of $r$ common factors, $B$ is the $N \times r$ matrix of factor loadings and $u_{t}$ is the idiosyncratic noise. Both the common factors and the idiosyncratic noises are allowed to exhibit (weak) cross and serial dynamics. So, the forecasts are computed from a factor augmented autoregression

$$
y_{t+1}=\alpha_{0}+\sum_{j=1}^{p} \alpha_{j} y_{t-j}+\psi^{\prime} F_{t}+e_{t+1},
$$

where $\psi=\left(\psi_{1}, \ldots, \psi_{r}\right)^{\prime}$. Thus, the forecaster gets the benefit of using all the predictors by using only a small set of factors. However, it is not clear that increasing the number of predictors is useful to improve the forecasts.

According to Stock and Watson (2011), the estimation of these models can be classified into three generations. The first generation of models are estimated using Gaussian maximum likelihood estimation and the Kalman filter. An advantage of this formulation is that it can handle data irregularities easily. However, the number of parameters to be estimated increases with the number of predictors, which complicates estimation when $N$ is large. Aruoba, Diebold, and Scotti (2009) and Camacho and Perez Quiros (2010) are recent examples.

When $N$ is sufficiently large, the second generation of models rely on nonparametric cross-sectional averaging methods, mainly based on principal components, to estimate the space spanned by the factors consistently. The result typically relies on the assumptions that the cross-correlation of the idiosyncratic components is weak and that the variability of the common component is not too small. In these cases, the common factors of the principal component estimator are consistent, as both $N$ and $T$ go to infinity. See, for instance, Stock and Watson (2002a) and Forni et al. (2000, 2005).

The third generation of models are based on hybrid methods that use consistent nonparametric estimates of the factors, which are then used as regressors to estimate the parameters of the state space representation. Finally, the model is written in state space form and the parameters are used in the Kalman filter to improve the efficiency of the 
estimation of the common factors. Doz, Giannone and Reichlin (2011) and Doz, Giannone and Reichlin (2012) are good references.

In practice, the number of predictors used to extract the factor varies considerably across applications. For example, the set of predictors used by Stock and Watson (2002b) and Forni et al. (2003) comprised 215 and 447 time series, respectively. Can it be possible that increasing $N$ does not improve the precision of the factor estimates? The literature about the number of series in dynamic factor models when they are used as forecasting tools is still scarce. Boivin and Ng (2006) find that factor-based forecasts extracted from 40 variables perform better than those extracted from 147 variables. Based on several simulations with principal component estimators, they suggest that the composition of the dataset as well as the properties of the idiosyncratic errors are crucial in determining the most convenient number of series. Using Kalman filter estimators, Alvarez, Camacho and Perez Quiros (2012) also relate the forecasting performance of the common factors forecasts to the composition of the dataset and to the persistence of the idiosyncratic component. Caggiano, Kapetanios and Labhard (2011), based on the principal component estimator, show that factors extracted from pre-screened series often yield satisfactory or even better results than using larger sets of series. Banbura and Runstler (2011), within the Kalman filter framework, find that around 30 variables are enough to estimate the common factors.

As regards the first generation of factor models, the Kalman filter provides expressions for the Mean Squared Error (MSE) for every time period and any number of series. In time-invariant systems, the filter reaches the steady state and the expression of the MSE of the estimator of the common factors no longer depends on time so it can be reduced by enlarging the number of predictors (see Peña and Poncela, 2004). Recently, Poncela and Ruiz (2012) provide a comprehensive analysis of how the uncertainty in the forecast, estimation and smoothness of the common factor is related to the number of series $N$. They conclude that around 30 series are enough to obtain common factors estimated with very low MSE, even in models with more than one common factor and serial dependence in the idiosyncratic noises. However, if the serial correlation in the idiosyncratic noises is very persistent, more variables might be needed to obtain the same MSEs, especially if 
the common factor is also highly persistent.

Assume that the vector of $N \times 1$ monthly indicators admits a single factor model decomposition and that the population parameters are known. Then, the vector of observed series is generated as

$$
x_{t}^{(N)}=B^{(N)} f_{t}+u_{t}^{(N)}
$$

where $B^{(N)}=\left(\beta_{1}, \ldots, \beta_{N}\right)^{\prime}$ is the $N \times 1$ loading vector, and $u_{t}^{(N)}$ is an $N \times 1$ vector of zero mean Gaussian white noise with finite and positive definite covariance matrix $R^{(N)}$, which might be non-diagonal. Notice that the the dependence on the number of series $N$ is explicitly stated through the superscript. The common factor follows an $\operatorname{AR}(1)$ as in (21) with $p_{f}=1$ and an autoregressive parameter $\phi_{11}$. Poncela and Ruiz (2012) show that the one-step-ahead steady-state MSE, given by the solution of the Riccati equation, is

$$
V(N)=\frac{\sigma_{f}^{2} T(N)-1+\phi_{11}^{2}+\sqrt{\left(\sigma_{f}^{2} T(N)-1+\phi_{11}^{2}\right)^{2}+4 \sigma_{f}^{2} T(N)}}{2 T(N)},
$$

where

$$
T(N)=B^{(N) \prime}\left(R^{(N)}\right)^{-1} B^{(N)} .
$$

The filtered steady-state MSE is given by

$$
W(N)=\frac{V(N)}{1+V(N) T(N)}
$$

and the smoothed steady-sated MSE is given by

$$
S(N)=\frac{V(N)\left(1+V(N) T(N)-\phi_{11}^{2}\right)}{(1+V(N) T(N))^{2}-\phi_{11}^{2}} .
$$

Note that the dependence on the number of series comes through $T(N)$, which becomes the sum of the signal to noise ratios, $T(N)=\sum_{i=1}^{N} \frac{\beta_{i}^{2}}{\sigma_{i}^{2}}$, when $R^{(N)}$ is diagonal. ${ }^{14}$

Poncela and Ruiz (2012) show that, adding an additional predictor, the filtered and the smoothed steady-state MSE typically decrease. They remain constant only if (i) the common factor is static, $\phi_{11}=0$; or (ii) the additional predictor is not informative, $\beta_{N+1}=0$ and its corresponding idiosyncratic noise, $u_{N+1}$, is not correlated with any of the predictors already included in the system.

\footnotetext{
${ }^{14}$ The result is also true for the nonstationary case, in which $\phi_{11}$ is allowed to reach 1.
} 
This framework helps to evaluate how many predictors lead to significant reductions in MSE. When the parameters of the models are known, the authors show that adding variables beyond a relatively small number (30 in the simulated experiments) does not decrease the filter uncertainty significantly. When the parameters must be estimated in empirical applications, using additional predictors leads to a trade off between the reductions in filter uncertainty and the increases in estimation uncertainty, which grows with the additional number of parameters that must be estimated, especially when the sample size is small. ${ }^{15}$ Using Monte Caro, the authors show that for sample sizes of $T=100$, the total uncertainty increases around $5 \%$ when the parameters of the model are estimated.

Notably, they show that the curve of the MSE against the number of series is Ushaped. Therefore, the uncertainty starts to increase beyond a certain number of variables. In particular, they find that the percentage of the parameter uncertainty over the total uncertainty is minimum when the number of variables is around 10 .

In the context of the second generation of factor models, Bai (2003) gives the expression for the asymptotic variance of the principal component estimator of the common factor. To check the finite sample performance of the estimator in empirical analyses, Boivin and $\mathrm{Ng}$ (2006) compute the statistic

$$
S_{f, f_{0}}=\frac{\operatorname{tr}\left(f_{0}^{\prime} \widehat{f}\left(\widehat{f}^{\prime} \hat{f}\right)^{-1} \widehat{f}^{\prime} f_{0}\right)}{\operatorname{tr}\left(f_{0}^{\prime}, f_{0}\right)}
$$

where $f_{0}$ and $\widehat{f}$ are $T \times r$ matrices of population factors and estimated common factors and $r$ is the number of factors. They simulate series according to the properties of the idiosyncratic noises and allow for heteroskedasticity and cross-correlation across categories. They check the effects of oversampling by extracting the factors from predictors that show very low factor loadings. Interestingly, they find average values of the statistic around 0.6, with a large associated dispersion that can go down to around 0.5 if oversampling occurs. They also find that it is useful to weight the data by their properties when constructing the factors. In addition, they find that oversampling, cross correlation and heteroskedasticity in the idiosyncratic noises deteriorate the forecasting performance. Using the database

\footnotetext{
${ }^{15}$ Note that the parameters uncertainty falls with the sample size $T$.
} 
suggested by Stock and Watson (2002b), Boivin and Ng (2006) show that factors extracted from as few as 40 pre-screened series often yield satisfactory or even better forecasting results than using all the series.

In an independent paper, Alvarez et al. (2012) examines the empirical relative forecasting performance at different horizons of factor models that belong to the first and the third generations by using both Monte Carlo exercises and US actual data. Since the data are collected from economic categories in the empirical economic applications, they evaluate the effects of cross-correlation across and within categories, the effects of dealing with oversampled categories and the effects of serial dependence of both the factors and the idiosyncratic components. Their results suggest that the forecasts from a factor model of the first generation that uses a set of preselected predictors outperform the forecasts from a factor model of the third generation that uses all the available predictors when the data exhibit the typical problems that characterized the empirical applications. Particularly, the gains appear when (i) the cross-correlation across the idiosyncratic errors within the same category is high, (ii) when there are oversampled categories and, especially, (iii) when the persistent of the common factor and/or the idiosyncratic components become moderately high.

\subsubsection{Forecasting using targeted predictors}

Although much professional attention has recently turned to big data approaches, the previous section states that bigger amounts of information are not necessarily better in forecasting accuracy. It now seems reasonable to think that the selection of indicators is a crucial step before building a model to forecast a variable of interest. However, the way in which the indicators are selected from a large set of potential candidates is still an open question and probably the source of intense debates in the near future.

In our opinion, the final set of indicators should exhibit the following characteristics, whose achievement must be used as a guidance in the selection procedure. First, the indicators must be updated in a timely manner and, therefore, they must be published before the variable of interest is released. Second, the indicators must be reliable. They should not be revised substantially after they are first published since the preliminary 
figures would incorporate noisy signals of the variable of interest.

Third, if one is interested in using the movements of the promptly available indicators as early signals of changes in the variable of interest, the indicators must be related to the variable of interest in the sense that they may cohere. Therefore, the factor extraction should not be independent of the target forecast. Some predictors might not be informative and the uncertainty of their coefficients in the factor extraction might not compensate their forecasting content.

Last, but not least, the final set of indicators obtained from selection procedures must be economically meaningful. It makes sense that the set of final indicators must comprise some representative indicators from all the economically relevant categories of data for the objective of forecasting a specific series, unless they are proven to be clearly useless in the model. In addition, it is hard to explain the selection of some disaggregated indicators when the total and most representative indicator of a category is skipped from the sample following pure statistical selection criteria.

Bai and Ng (2008) propose several methods to perform forecasts from targeted predictors. The first method simply uses regressions of the target over each predictor and select those with $t$-statistics over a certain threshold. Then, the principal component estimator is applied over the selected predictors. One of the main drawbacks of this selection procedure is that it does not consider the correlation across the predictors. A trivial but illustrative example is the case that an informative predictor is, by mistake, saved twice in the database. In this case, the uninformative predictor would be selected twice.

To perform principal components over a reduced number of predictors, these authors also apply other subset selection methods that are based on modified least squares. Skipping details, the methods rely on the minimization problem of the sum of squared residuals of the regression of the target variable on all the available regressors, which is modified by different penalty functions. The Ridge Regression (RR) estimator uses the sum of the squared parameters, the Least Absolute Shrinkage and Selection Operator (LASSO) estimator uses the sum of the absolute value and the Elastic Net (EN) estimator uses a combination of these two approaches. Based on the previous methods, one can obtain an ordering of the predictors taking into account the penalty. Then, principal components is 
performed over the top ranked predictors. Using a database of 132 predictors, they show important reductions (up to one half) in inflation forecast errors from targeted predictions over the principal component forecasts that does not perform targeting at all. The gains are more evident when the forecasting horizon increases.

Fuentes, Poncela and Rodriguez (2012) develop a forecasting method that combines the Partial Least Squares (PLS) estimator, where the factors are formed from an eigenvalue decomposition that captures the covariance between the predictors and the target, with the targeted predictors framework, which sets the parameters of uninformative predictors to zero by using penalty functions in the optimization procedure. ${ }^{16}$ Using the database of Bai and Ng (2008), they show that the inflation forecasts of the Sparse PLS estimator outperform the forecasts of principal component estimators even in short forecasting horizons.

To reduce the number of predictors used in the principal component estimator, Boivin and $\mathrm{Ng}$ (2006) focus on the view that problems of this method tend to arise when the idiosyncratic errors are cross-correlated. Therefore, they hey propose removing the time series with highest correlation of idiosyncratic components. This selection procedure leads to a final set of 40 predictors often yield satisfactory or even better forecasting results than using from an initial set of 147 series.

Alvarez et al. (2012) show that focusing on the correlation of idiosyncratic components typically leads to final sets of predictors with low economic content. In particular, the method selects disaggregated concepts from each category instead of the headline concepts. Sometimes, some economically interesting categories are not represented and some others become oversampled. To overcome this drawback, they select one representative variable of each category as the variable with highest correlation within the indicators of the same category. In practice, they find that the selected indicator of each category typically coincides with the aggregate headline concept, which converts the proposal in an economically meaningful selection criterion. Alvarez et al. (2012) show that this selection method leads to significant forecasting improvements with respect to the statistical method proposed by Boivin and Ng (2006).

\footnotetext{
${ }^{16}$ Examples of PLS estimators appear in Groen and Kapetanios (2008) and Kelly and Pruitt (2012).
} 


\subsection{Markov-switching factor models}

Diebold and Rudebusch (1996) were the first to suggest a unified model that captures the notions of comovements and asymmetries that characterize the business cycle features from a set of economic indicators. Comovements are captured with a dynamic factor model as in Stock and Watson (1991) while asymmetries are modelled by using the Markovswitching specification advocated by Hamilton (1989). Kim and Yoo (1995), Chauvet (1998) and Kim and Nelson (1998) integrate the two features in a single Markov-switching dynamic factor model (MS-DFM). Camacho, Perez Quiros and Poncela (2012a) find that the fully non-linear multivariate specification outperforms the "shortcut" of using a linear factor model to obtain a coincident indicator, which is then used to compute the Markovswitching probabilities.

To account for the business cycle asymmetries, these models assume that the dynamic behavior of the factor is governed by an unobserved regime-switching state variable, $s_{t}$. Accordingly, the dynamics of the factor is extended to account for nonlinearities as follows:

$$
f_{t}=\mu_{s t}+\phi_{f 1} f_{t-1}+\ldots+\phi_{f p_{f}} f_{t-p_{f}}+\epsilon_{f t}
$$

where $\epsilon_{f t} \sim i i N\left(0, \sigma_{f}^{2}\right) \cdot{ }^{17}$ Within this framework, one can label $s_{t}=0$ and $s_{t}=1$ as the expansion and recession states at time $t$. In addition, it is standard to assume that the state variable evolves according to an irreducible 2-state Markov chain whose transition probabilities are defined by

$$
p\left(s_{t}=j \mid s_{t-1}=i, s_{t-2}=h, \ldots, I_{t-1}\right)=p\left(s_{t}=j \mid s_{t-1}=i\right)=p_{i j},
$$

where $i, j=0,1$ and $I_{t}$ is the information set up to period $t$.

The particular form of the state space representation for the switching dynamic factor comes from subtle modifications of the state space specification of the linear model. Under the simplifying assumptions used in the linear factor model, the nonlinear state space form only requires adding

$$
\left(\mu_{s_{t}}, 0,0,0,0,0,0,0,0,0,0\right)
$$

\footnotetext{
${ }^{17}$ The dynamics can be adapted to account for regime shifts in the autoregressive parameters and in the variance. In addition, the nonlinearities can be assumed in the mean or in the drift of the factor.
} 
to the linear measurement equation.

It is worth mentioning that both the measurement and the transition equations could be regime switching. To handle this nonlinear dynamics, Kim (1994) proposes a useful nonlinear discrete version of the Kalman filter which is combined with Hamilton's nonlinear filter in one algorithm. However, each iteration of the Kalman filter produces a twofold increase in the number of cases to consider, which would become computationally unfeasible since it would lead to $2^{T}$ number of states to consider in the likelihood function. Kim (1994) proposes an approximation that consist of a weighted average of the updating procedures by the probabilities of the Markov state, in which the mixture of Gaussian densities is collapsed after each observation. ${ }^{18}$

The main advantage of these nonlinear models is that they convert the information contained in the economic indicators about the business cycle into inferences of the probabilities of recession. Hence, they provide economic agents with statistical definitions of some terms usually employed in business cycle analysis, such as green shoots, double-dip recessions, recoveries or turning points, which, in this context, are very easy to interpret and can be timelily and automatically updated. Needless is to say, the probabilities of recession computed from a statistical model are transparent, objective and free of units of measurement, which facilitates international business cycle comparisons.

Although some recent empirical proposals try to examine the empirical reliability of these models in computing real-time inferences of the US business cycle states (see Chauvet and Hamilton, 2006, Chauvet and Piger, 2008, and Hamilton, 2011), the analyses are not developed in actual real time. The original MS-DFM was originally designed to deal with balanced panels of business cycle indicators so it could not handle the typical problems of the day-to-day monitoring of economic activity: mixed frequencies and ragged ends.

To overcome these limitations, Camacho, Perez-Quiros and Poncela (2012b) adapt the MS-DFM to permit whatever business cycle economic indicator, regardless of publication delays and frequency. Based on the techniques described in the linear context to handle missing data, their procedure deals with missing observations by using a time-varying

\footnotetext{
${ }^{18}$ Kim and Nelson (1998) use an alterative approach which is based on Gibbs sampling by casting the model in a Bayesian framework.
} 
nonlinear Kalman filter. Whenever the data is not observed, the missing observations are replaced by random draws from a variable whose distribution cannot depend on the parameter space that characterizes the Kalman filter or the Markov-switching chain. The corresponding row is then skipped in the Kalman recursion and the measurement equation for the missing observation is set to the random choice.

The analyses developed in an actual real-time scenario by Camacho, et al. (2012b) suggest that MS-DFM identifies the NBER turning point dates in real time with reasonable accuracy. Accordingly, this model appears to be a reasonable framework to track the US business cycle in real time. In addition, Camacho et al. (2013) show the usefulness of MS-DFM to obtain an indicator of the overall economic activity and to compute business cycle inferences in the euro area. Their findings indicate that the model exhibits a remarkably ability to track the CEPR (Center for Economic Policy Research) Business Cycle Dating Committee chronology as captured in the state probabilities for the overall economic indicator. However, MS-DFM provides improvements over the NBER and the CEPR in the timeliness with which they identify business cycle turning points.

\subsection{A critical overview}

Essentially, MIDAS and bridge equation models are both based on single-equation models, which are usually enlarged with auxiliary regressions to update the forecasts of the explanatory variables when computing iterative forecasts. By contrast, MF-VAR and factor models are multivariate specifications and can compute forecasts of the individual indicators. This is of special interest when the analysis of the economic developments focuses not only on forecasting by how much the variable of interest is revised as new information become available, but also on assessing the revisions. In other words, modeling the joint dynamics of the higher-frequency indicators and the lower-frequency variables in a unified framework facilitates the analysis of the relative impact of each of the arrivals on the variable of interest.

MF-VAR and factor models are model-driven as they are cast in state space forms and estimated by using the Kalman filter. However, MIDAS and bridge equations are observation-driven as they are formulated exclusively in terms of observable data and do 
not involve latent processes, which avoids the need to formulate measurement equations and filtering. In addition, MIDAS models are very parsimonious since the lag polynomials are represented as functions of a very reduced number of parameters. Therefore, they require less parameters and they are also computationally much less demanding, especially when using very high frequency indicators.

State-space models can be quite appealing, as one explicitly sets the specific dynamics for all the series involved, the high-frequency data series, the latent high-frequency series treated as missing and the low-frequency observed processes, and their respective relationships. This enables the forecasters to evaluate the information content of the arriving information. In addition, they are suitable representations to account for the specific aggregation equations that comes from the handling flow data at different frequencies.

The models reviewed in this paper establish that although some series could be integrated they are not cointegrated. Clements and Hendry (1995) analyze the need to incorporate the cointegration relations in forecasting with VAR models. However, the general implication of cointegration for forecasting in the wide spectra of models covered in this paper is still not available. Very recently, Banerjee, Marcellino and Masten (2013) have analyzed the advantages in forecasting with Factor-Augmented Error Correction Models which combine error-correction, cointegration and dynamic factor models. Goetz, Hecq and Urbain (2012) have proposed forecasting models that incorporate cointegration relations within the MIDAS framework. Alternatively, the nonstationary common trends can be modelled. In the latter case, Peña and Poncela (2004) show that the gain in precision, in terms of the prediction MSE, of small nonstationary factor models with respect to univariate ARIMA and pooled forecasts depends on the common information and increases with the number of time series and the sum of the relative sizes of the factor loadings. Fuleky and Bonham (2013) analyze empirically the forecasting performance of small mixed frequency factor models when the observed variables share stochastic trends.

One alternative to be pursued is to consider the combination of the forecasts from some or all the models described in this section, as in Diron (2008), Kuzin at al. (2011) or Timmermann (2006). Although this is beyond the scope of this paper, there are a number of open questions in the empirical application of pooling for short term forecasting. 
Examples are how to determine the number of models involved in the combination and how to perform the combinations in real-time since it may lead to multiple forecasts when forecasting in changing environments.

This discussion reveals that it is difficult to rank all the alternative forecasting approaches purely on the basis of theoretical considerations. Their relative merits will depend on the specific empirical analysis to be developed. Therefore, their performances are better assessed in specific economic applications.

Finally, after reviewing all these forecasting techniques, it is convenient to point out that all these "engineering type" models that we have surveyed are algorithms that try to mimic the way in which business cycle professional forecasters update their views of the economy by incorporating newly release of information. In this sense, these are not "structural" models that try to capture casual relations. These are reduced form models that are designed to be automatically updated to predict the variable of interest by exploiting just cross-correlations or non-linear comovements.

\section{An empirical example}

The purpose of this section is to examine the real-time forecasting performance of US GDP in a horse race of the models described below which use as input several monthly economic indicators that provide early signals of GDP changes. Of course, the selection of the set of indicators is not trivial and a source of debate. To this end, we benefit from the selection of indicators developed by the NBER's Business Cycle Dating Committee to maintain the chronology of the U.S. business cycle. The Committee acknowledges on its website that it applies its judgment based on the behavior of various measures of broad activity: real GDP, employment, real income, sales and industrial production.

According to the Committee's criterion, we use a dataset that comprises, apart from real GDP, four monthly indicators: industrial production index, nonfarm payroll employment, personal income less transfer payments and real manufacturing and trade sales. A similar set of monthly indicators has extensively be used in the literature to compute coincident indexes of the US economic activity as the composite index of coincident indicators 
released by the Conference Board, the old Stock-Watson Experimental Coincident Index (see Stock and Watson, 1991). Recently, several empirical analyses have been developed by enlarging the set of monthly indicators with quarterly GDP, as in Mariano and Murasawa (2003, 2010), Aruoba and Diebold (2010), Camacho et al. (2012a, 2012b) and Camacho (2013).

To perform a realistic assessment of the actual empirical reliability of the competing models, we evaluate their forecasting performance through a data set that consists of the vintages available at the time of each forecast. In particular, the forecasts are computed from recursively estimated models that use the information available in the middle of each month $t$ over a sample of 13 years that covers the period December, 1998 to January, 2011. Therefore, the real-time analysis does not include the data revisions that were not available at the time the model would have been used and has to manage with incomplete data sets at the time of each inference.

To clarify understanding, let us describe the publication calendar of the economic indicators used in the real-time analysis. At the end of month $t$, Industrial Production is published on the 15th of the month $t+1$; Non-farm Employees is published on the 8 th of the month $t+1$, Real Personal Income is published on the 27th of the month $t+1$, Real Manufacturing and Trade Sales is published on the 27th of the month $t+2,{ }^{19}$ and GDP is published on the 15 th of $t+2$, whenever $t$ is March, June, September or December. To simplify the real-time analysis, we consider that the forecasts are computed on the 15 th of each month, where employment and industrial production are available for the previous month.

According to the interest of business people in following the real-time developments of the current economic activity, we focus on the forecasts for the GDP growth of a given quarter that are computed during the quarter and after the quarter. To understand how the sequence of forecasts works in practice, based on different sets of monthly information, let us examine the stylized example of data releases depicted in Figure 1. The next three forecasts computed of GDP growth of quarter Q corresponds to the forecasts computed from the information set that is available in the first, second and third months of this

\footnotetext{
${ }^{19}$ The nominal indicator is published on the 14 th of $t+2$.
} 
quarter, respectively. Therefore, they are labeled as first, second and third nowcasts of GDP growth of quarter Q. Since the GDP figure for this quarter is not available until about the second month of quarter $\mathrm{Q}+1$, we perform the last forecast of GDP growth of quarter $\mathrm{Q}$ in the first month of quarter $\mathrm{Q}+1$, which we take as the ending point to produce forecasts for this quarter. We denote this forecast as the backcast of quarter $\mathrm{Q}$ to emphasize the fact that it refers to a prediction of the past.

All the series are seasonally adjusted. To make them stationary, we take the first difference of the natural log of each series and multiply it by 100 , which is approximately equal to the quarterly or monthly percentage growth rate. Bridge equations, MF-VAR and factor models are estimated by maximum likelihood while MIDAS models are estimated by nonlinear least squares.

Forecasts from bridge equations and MIDAS models come from multivariate specifications, where the four monthly indicators and one lag of GDP growth are included in the right-hand-side specifications described above. Bridge equations are estimated with a maximum lag length of 2 lags for quarterly variable and 12 lags of monthly indicators. In the MIDAS specifications, the parameters of the exponential lag function are restricted to $\theta_{1}<5$ and $\theta_{2}<0$ and the maximum number of lags chosen is $K=12$ months, following Kuzin et al. (2011). Concerning the MF-VAR model, the maximum the lag order is $p=2$ in order to ensure that the number of parameters to be estimated within the model is feasible.

With respect to the linear factor model specification, we focus exclusively on the smallscale dynamic factor models, although acknowledge that comparisons of forecasts from large-scale factor models some of the alternative approaches described in this paper would clearly be of interest. The maximum lag length of the autoregressive processes are set to $p_{f}=p_{j}=2, j=0,1, \ldots, 4$. Before estimating the model, the variables are standardized to have a zero mean and a variance equal to one. Therefore, final forecasts are recursively computed by multiplying initial forecasts of the model by the standard deviation, and then adding the means, which are also recursively recomputed. Apart from these assumptions, in the Markov-switching specification we follow Camacho and Perez Quiros (2007) and assume that $p_{f}=0$ so all the common dynamics come from the switch from one state to 
the other, while linear serial correlation is added through the idiosyncratic noises. ${ }^{20}$

In the empirical literature that compares some of the models outlined above, the authors find no clear winner in terms of forecasting performance. Bai, Ghysels, and Wright (2011) examine the relationship between MIDAS regressions and state-space models applied to mixed-frequency data and conclude that Kalman filter forecasts are typically a little better, but MIDAS regressions can be more accurate if the state-space model is misspecified or over-parameterized. Kuzin, Marcellino, and Schumacher (2011) compare the accuracy of Euro Area GDP growth forecasts from MIDAS regressions and MF-VARs estimated by maximum likelihood and find that the relative performances of these models differ depending on the predictors and forecast horizons.

Table 1 reports the performance of the model to forecast US GDP growth in real time. In particular, the table shows the mean squared errors, which are computed by as averaged deviations of the real-time (1998.1 an ending in 2011.01) forecasts from the finally revised GDP growth figures. According to the forecast experiment design, nowcasting refers to the forecasts computed within the reference quarter and backcasting refers to the forecast computed one month after the end of the reference quarter.

The most relevant result that derives from the table is that there is a major gain in forecasting using indicators, no matter which model is used. A simple autoregressive model is notably improved by all the models considered in this survey. The old story that the autoregressive models are difficult to beat, does not hold when using real time available indicators which contain reliable information about the current activity. In addition, another result that is common to all the models is that the upcoming information from the monthly indicators is important to improve upon the forecasting accuracy in real time. The table reports that, for all the specifications, the forecast uncertainty falls drastically as more information becomes available when computing the forecasts. All the models exhibit much lower averaged mistakes when performing the GDP growth backcasts with respect to the nowcasts because almost all the monthly information relative to the reference quarter is available at the time of computing the backcasts.

The relative performance of one model versus the others is more difficult to distin-

\footnotetext{
${ }^{20}$ Note that this assumption avoids identification problems.
} 
guish, because the gains from one model to another are only marginal, although the factor models and the VAR have the advantage of allowing a joint forecast of all the variables of interest. In addition, the nonlinear approach offers the interesting additional information of converting the business cycle signals provided by the economic indicators into recession probabilities. Figure 4 plots the values of the real-time recession probabilities in each period $t$, which are inferred by using the latest vintage. What it is clear, as a lesson to practitioners, is that it is always better to forecast with models that incorporate updated information of current activity. The decision of which model to use depends on the additional information that the practitioner wants to produce.

\section{Conclusion}

The Great Recession has led to renewed scientific interest in short-term forecasting. Nowadays, the monitoring of the real-time economic developments through some key economic aggregates is a plentiful source of debate. How to deal with the lack of information hindering timely publication of macroeconomic variables, how to fill in the missing values when the time series exhibit different frequencies, how to use the economic aggregates with short time spans, and how many and which kind of variables should be included in the forecasting models are still open questions.

The traditional literature and methodology on macroeconomic forecasting usually assumed either that all processes were sampled at the same frequency and that their updates were available at the same time, or accommodated the time series accordingly. However, some key macroeconomic variables for Central Banks, Governments and private research institutions, such as GDP, are unavailable at frequencies higher than quarterly. In addition, the publication calendar of the national statistical agencies are hardly often synchronized, which, added to the publication lags that characterize the data flow, requires the forecast model to be able to deal with unobservable data at the end of the data vintages.

This paper reviews the recent developments on short-term forecasting by trying to address all the practitioners' concerns on the day-to-day analysis of the economic developments which means dealing with these real-time forecasting problems. In particular, 
we perform a critical overview of bridge equations, MIDAS models, mixed-frequency VAR specification and linear and nonlinear factor models.

It is difficult to rank the short-term forecasting approaches purely on the basis of theoretical considerations. Ultimately, their relative merits will depend on their success in forecasting the variable of interest. Therefore, we believe that their performances are better assessed in specific economic applications. For this purpose, we establish the overall realtime forecasting performance of these models to forecast US GDP growth rate from a set of monthly indicators that include industrial production, employment income and sales from January 1998, and ending in January 2011. Notably, the forecasts are computed recursively using only information available at the time the forecasts are made. We find that the Markov-switching dynamic factor model outperforms the alternatives. Additionally, we check that this model is able to infer the US business cycle in real time, with notable accuracy.

The short-term forecasting approaches reviewed here can be extended in several potentially fruitful directions, including, but not limited to, the following. First, it will be of interest to attempt to include indicators beyond macroeconomic and financial data, such as headline news, social networks and searches that take place over the internet. Second, due to recent advances in information technologies, data are becoming increasingly available with unprecedented complexity and degree of disaggregation. Since more data are not necessarily better for empirical forecasting, the problem of systematically selecting some key indicators from the huge set of potentially available indicators deserves further studies from both theoretical and empirical perspectives. Third, although combining forecasts from the alternative models could yield sizeable gains, the huge number of potential combinations and different ways to perform the forecast combinations precluded us from exploring this possibility in the paper. Finally, exploring the consequences of incorporating cointegration relations in all the different forecasting specifications could also be a line for future research. 


\section{References}

[1] Altissimo, F., Cristadoro, R., Forni, M., Lippi, M., and Veronese, G. 2010. New Eurocoin: Tracking economic growth in real time. Review of Economics and Statistics 92:1024-1034.

[2] Alvarez, R., Camacho, M. and Perez-Quiros, G. 2012. Finite sample performance of small versus large scale dynamic factor models. CEPR Working Paper No. 8867.

[3] Andreou, E., Ghysels, E., and Kourtellos, A. 2011 Forecasting with mixed-frequency data. In M. Clements and D. Hendry, eds., The Oxford Handbook of Economic Forecasting, Oxford: Oxford University Press.

[4] Angelini, E., Henry, J., and Marcellino, M. 2006. Interpolation and backdating with a large information set. Journal of Economic Dynamics and Control 30: 2693-2724.

[5] Armesto, M., Engemann, K. and Owyang, M. 2010. Forecasting with mixed frequencies. Federal Reserve Bank of St. Louis Review 92, 521-536.

[6] Artis, M., Marcelino, M. and Proietti, T. 2004. Dating business cycle: a methodological contribution with an application to the euro area. Oxford Bulletin of Economics and Statistics 66:537-565.

[7] Aruoba, B., Diebold, F., and Scotti, C. 2009. Real-time measurement of business conditions. Journal of Business and Economic Statistics 27: 417-427.

[8] Aruoba, B., and Diebold, F. 2010. Real-time macroeconomic monitoring: Real activity, inflation, and interactions. American Economic Review: Papers and Proceedings 100: $20-24$.

[9] Bai, J. 2003. Inferential theory for factor models of large dimensions. Econometrica $71: 135-171$.

[10] Bai, J., and Ng, S. 2008. Forecasting economic time series using targeted predictors. Journal of Econometrics 146: 304-317. 
[11] Bai, J., and Ng., S. 2008. Large dimensional factor analysis. Foundations and Trends in Econometrics 3: 89-163.

[12] Bai, J., Ghysels, E., and Wright, J. 2012. State space models and midas regressions. Econometric Reviews, forthcoming.

[13] Baffigi, A., Golinelli, R., and Parigi, G. 2004. Bridge models to forecast the Euro area GDP. International Journal of Forecasting 20: 447-446.

[14] Banerjee A., Marcellino, M. and Masten, I. 2008.Forecsting with Factor Augmented Error Correction Models. International Journal of Forecasting forthcoming.

[15] Banbura, M., and Runstler, G. 2011. A look into the factor model black box: publication lags and the role of hard and soft data in forecasting GDP. International Journal of Forecasting 27:333-346.

[16] Banbura, M., Giannone, D., and Reichlin, L. 2011. Nowcasting. In M. Clements and D. Hendry, eds., Oxford Handbook on Economic Forecasting, Oxford University Press.

[17] Banbura, M., Giannone, D., Modugno, M., and Reichlin, L. 2013. Now-casting and the real-time data flow. In G. Elliott and A. Timmermann, eds., Handbook of Economic Forecasting, Volume 2, Elsevier-North Holland.

[18] dal Bianco, M., Camacho, M., and Perez Quiros, G. 2012. Short-run forecasting of the euro-dollar exchange rates with economic fundamentals. Journal of International Money and Finance 31: 377-396.

[19] Boivin, J., and Ng, S. 2006 Are more data always better for factor analysis? Journal of Econometrics 132: 169-194.

[20] Breitung, J. and I. Choi 2013. Factor Models in EE Handbook of Research Methods and Applications in Empirical Macroeconomics, edited by N. Hashimzade and M. Thornton, Edward Elgar.

[21] Caggiano, G., Kapetanios G., and Labhard, V. 2011. Are more data always better for factor analysis? Results from the euro area, the six largest euro area countries and the UK. Journal of Forecasting 30: 736-752. 
[22] Camacho, M. 2013. Mixed-frequency VAR models with Markov-switching dynamics. Economics Letters, forthcoming.

[23] Camacho, M., and Perez Quiros, G. 2007. Jump-and-rest effect of U.S. business cycles. Studies in Nonlinear Dynamics and Econometrics 11(4): article 3.

[24] Camacho, M., and Perez Quiros, G. 2010. Introducing the EURO-STING: Short Term INdicator of Euro Area Growth. Journal of Applied Econometrics 25: 663-694.

[25] Camacho, M., and Perez Quiros, G. 2011. Spain-STING: Spain Short Term INdicator of Growth. The Manchester School 79: 594-616.

[26] Camacho, M., and Domenech, R. 2012. MICA-BBVA: A factor model of economic and financial indicators for short-term GDP forecasting. SERIES, Journal of the Spanish Economic Association3: 475-497.

[27] Camacho, M., Perez Quiros, G., and Poncela, P. 2012a. Extracting nonlinear signals from several economic indicators. CEPR Working Paper No. 8865.

[28] Camacho, M., Perez Quiros, G., and Poncela, P. 2012b. Markov-switching dynamic factor models in real time. CEPR Working Paper No. 8866.

[29] Camacho, M., Perez Quiros, G., and Poncela, P. 2013. Green shoots and double dips in the Euro area. A real time measure. International Journal of Forecasting, forthcoming.

[30] Chauvet, M. 1998. An econometric characterization of business cycle dynamics with factor structure and regime switches. International Economic Review 39: 969-96.

[31] Chauvet, M., and Hamilton, J. 2006. Dating business cycle turning points in real time. In Nonlinear Time Series Analysis of Business Cycles, eds. C. Milas, P. Rothman, and D. Van Dijk. Amsterdam: Elsevier Science, pp. 1-54.

[32] Chauvet, M., and Piger, J. 2008. A comparison of the real-time performance of business cycle dating methods. Journal of Business and Economic Statistics 26: 42-49. 
[33] Clements, M., and Galvao, B. 2008. Macroeconomic forecasting with mixed-frequency data: Forecasting output growth in the United States. Journal of Business and Economic Statistics 26: 546-554.

[34] Clements, M., and Galvao, B. 2009. Forecasting US output growth using leading indicators: an appraisal using MIDAS models. Journal of Applied Econometrics 24: 1187-1206.

[35] Clements M.P. and Hendry D.F. 1998. Forecasting Economic Time Series. Cambridge. Cambridge University Press.

[36] Clements M.P. and Hendry, D.F. 1995. Forecasting in Cointegrated Systems. Journal of Applied Econometrics 10: 127-146

[37] Denton, Frank T., and Kuiper, J. The effect of measurement errors on parameter estimates and forecasts: A case study based on the Canadian preliminary National Accounts. Review of Economics and Statistics 47: 198-206.

[38] Diebold, F. 1998. Elements of forecasting. South-Western College Pub.

[39] Diebold, F., and Rudebusch, G. 1991. Forecasting output with the composite leading index: A real-time analysis. Joumal of the American Statistical Association 86: 603610.

[40] Diron, M. 2008. Short-term forecasts of Euro Area real GDP growth. An assessment of real-time performance based on vintage data. Journal of Forecasting 27: 371-390.

[41] Doz, C., Giannone, D., and Reichlin, L. 2011. A two-step estimator for large approximate dynamic factor models based on Kalman filtering. Journal of Econometrics 164: 188-205.

[42] Doz, C., Giannone, D., and Reichlin, L. 2012. A Quasi Maximum Likelihood approach for large approximate dynamic factor models. Review of Economics and Statistics 94:1014-1024. 
[43] Forni, M., Hallin, M., Lippi, M., and Reichlin, L. 2000. The generalized dynamicfactor model: identification and estimation. Review of Economics and Statistics 82: $540-554$.

[44] Forni, M., Hallin, M., Lippi, M., and Reichlin, L. 2003. Do financial variables help forecasting inflation and real activity in the euro area?', Journal of Monetary Economics 50: 1243-1255.

[45] Forni, M., Hallin, M., Lippi, M., and Reichlin, L. 2005. The generalized dynamic factor model: one sided estimation and forecasting. Journal of the American Statistical Association 100: 830-840.

[46] Foroni, C. and Marcellino, M. 2013. A survey of econometric methods for mixedfrequency data. Norges Bank Working Paper 2013-6.

[47] Fuentes, J., Poncela, P. and Rodriguez, J. 2012. Sparse PLS in Time Series for Macroeconomic Forecasting. Working Paper 12-22. Statistics and Econometrics Series 16. Universidad Carlos III de Madrid.

[48] Fuleky, P. and C. Bonham. 2013. Forecasting Based on Common Trends in Mixed Frequency Samples. Macroeconomic Dynamics (forthcoming).

[49] Galvao , A. 2013. Changes in predictive ability with mixed frequency data. International Journal of Forecasting 29: 395-410.

[50] Ghysels, E. 2012. Macroeconomics and the reality of mixed frequency data. University of North Carolina at Chapel Hill, Mimeo.

[51] Ghysels, E., Santa Clara, P., and Valkanov, R. 2002. The MIDAS touch: Mixed data sampling regression models. Working paper, UNC and UCLA.

[52] Ghysels, E., Sinko, A., and Valkanov, R. 2007. MIDAS regressions: further results and new directions. Econometric Reviews 26: 53-90.

[53] Ghysels, E., and Valkanov, R. 2012, Forecasting volatility with MIDAS. In L. Bauwens, C. Hafner, and S. Laurent, eds., Handbook of Volatility Models and Their Applications. Wiley. 
[54] Goetz, T., Hecq, A., and Urbain, J. 2012. Forecasting mixed frequency time series with ecm-midas models. WP Maastricht University.

[55] Groen, J. and Kapetanios, G. 2008. Revisiting useful approaches to data-rich macroeconomic forecasting. Federal Reserve Bank of New York Staff Report 327.

[56] Guerin, P. and Marcellino, M. 2013. Markov switching MIDAS models. Journal of Business and Economic Statistics 31: 45-56.

[57] Gupta, R., and Kabundi, A. 2011. A large factor model for forecasting macroeconomic variables in South Africa. International Journal of Forecasting 27: 1076-1088.

[58] Hamilton, J. 1989. A new approach to the economic analysis of nonstationary time series and the business cycles. Econometrica 57: 357-384.

[59] Hendry, D. F. and Mizon G.E. (2012) Unpredictability in economic analysis, econometric modelling and forecasting. Forthcoming Journal of Econometrics.

[60] Ingenito, R. and Trehan, B. 1996. Using monthly data to predict quarterly output. Federal Reserve Bank of San Francisco Economic Review, 3.

[61] Kelly, B., and S. Pruitt. 2012. The three-pass regression filter: A new approach to forecasting using many predictors. Chicago Booth Paper No. 11-19.

[62] Kim, C., and Nelson, C. 1998. Business cycle turning points, a new coincident index, and tests of duration dependence based on a dynamic factor model with regime switching. Review of Economics and Statistics 80: 188-201.

[63] Kim, C., and Yoo, J.S. 1995. New index of coincident indicators: A multivariate Markov switching factor model approach. Journal of Monetary Economics 36: 607630.

[64] Koenig, E., Dolmas, S., and Piger, J. 2003. The use and abuse of real-time data in economic forecasting. Review of Economics and Statistics 85: 618-628. 
[65] Kuzin, V., Marcellino, M., and Schumacher, Ch., 2012. MIDAS vs. mixed-frequency VAR: Nowcasting GDP in the euro area. International Journal of Forecasting 27: 529-542.

[66] Marcellino, M., and Schumacher, C. 2010. Factor-MIDAS for now- and forecasting with ragged-edge data: A model comparison for German GDP. Oxford Bulletin of Economics and Statistics 72: 518-550.

[67] Marcellino, M., Stock, J., and Watson, M. 2006. A comparison of direct and iterated multistep AR methods for forecasting macroeconomic time series. Journal of Econometrics 135: 499-526.

[68] Mariano, R., and Murasawa, Y. 2003. A new coincident index os business cycles based on monthly and quarterly series. Journal of Applied Econometrics 18: 427-443.

[69] Mariano, R., and Murasawa, Y. 2010. A coincident index, common Factors, and monthly real GDP. Oxford Bulletin of Economics and Statistics 72: 27-46.

[70] Molodtsova, T., Nikolsko-Rzhevskyy A., and Papell, D. 2008. Taylor rules with realtime data: A tale of two countries and one exchange rate. Journal of Monetary Economics 55: S73-S79.

[71] Nunes, L. 2005. Nowcasting quarterly GDP growth in a monthly coincident indicator model. Journal of Forecasting 24: 575-592.

[72] Peña, D., and Poncela, P. 2004. Forecasting with nonstationary dynamic factor models. Journal of Econometrics 119: 291-321.

[73] Pfeffermann, D. and Tiller, R. 2005. Bootstrap approximation to prediction MSE for state-space models with estimated parameters. Journal of Time Series Analysis 26, 893-916.

[74] Poncela, P. and Ruiz, E. 2012. More is not always better: back to the Kalman filter in Dynamic Factor Models. Working Paper 12-23. Universidad Carlos III de Madrid. 
[75] Rodriguez, A. and Ruiz, E. 2009. Bootstrap Prediction Intervals in State Space Models. Journal of Time Series Analysis 30, 167-178.

[76] Rojas, A.J. 2011. On the discrete-time algebraic Riccati equation and its solution in closed-form. Preprints of the 18th IFAC World Congress, Milan.

[77] Runstler, G., and Sedillot, F. 2003. Short-term estimates of euro area real GDP by means of monthly data. ECB Working Paper no. 276.

[78] Stark, T., and Croushore, D. 2002. Forecasting with a real-time data set for macroeconomists. Journal of Macroeconomics 24: 507-531.

[79] Stock J., and Watson, M. 2002a. Forecasting using Principal Components from a large number of predictors. Journal of the American Statistical Association 97: 1167-79.

[80] Stock J., and Watson, M. 2002b. Macroeconomic Forecasting Using Diffusion Indexes. Journal of Business and Economic Statistics 20: 147-162.

[81] Stock J., and Watson, M. 2006. Forecasting with many predictors. In G. Elliot, C. Granger, and A. Timmermann (eds.), Handbook of Economic Forecasting, vol.1, Elsevier.

[82] Stock, J., and Watson, M. 2002. Macroeconomic forecasting using diffusion indexes. Journal of Business and Economic Statistics 20: 147-162.

[83] Stock, J., and Watson, M. 2011. Dynamic factor models. In M. Clements and D. Hendry, eds., The Oxford Handbook of Economic Forecasting, Oxford: Oxford University Press.

[84] Timmerman, A. 2006. Forecast Combinations in Handbook of Economic Forecasting, ed. G. Elliott, C. W. J. Granger, and A. Timmermann. Amsterdam: Elsevier, NorthHolland, 2006

[85] Wall, K. D. and Stoffer, D. S. 2002. A state space approach to bootstrapping conditional forecasts in ARMA models. Journal of Time Series Analysis 23, 733-51. 
[86] West, K. D. 2006. Forecast Evaluation. Handbook of Economic Forecasting, ed. G. Elliott, C. W. J. Granger, and A. Timmermann. Amsterdam: Elsevier, North-Holland, 2006

[87] Zadrozny, P. 1990. Estimating a multivariate ARMA model with mixed-frequency data: An application to forecasting U.S. GNP at monthly intervals. Federal Reserve Bank of Atlanta Working Paper Series No. 6. 
Table 1. Real-time forecasting accuracy

\begin{tabular}{lcccccc}
\hline & Bridge & MIDAS & MF-VAR & DFM & MS-DFM & AR \\
\hline Nowcasting & 0.341 & 0.379 & 0.387 & 0.328 & 0.325 & 0.416 \\
Backcasting & 0.232 & 0.257 & 0.302 & 0.234 & 0.224 & 0.416 \\
\hline
\end{tabular}

Notes. The table shows the mean squared errors, which are computed by as averaged deviations of the real-time (1976.12 an ending in 2011.01) forecasts from the finally revised GDP growth figures. Nowcasting refers to the forecasts computed within the reference quarter and backcasting refers to the forecast computed one month after the end of the reference quarter. 
Figure 1. Forecast timeline

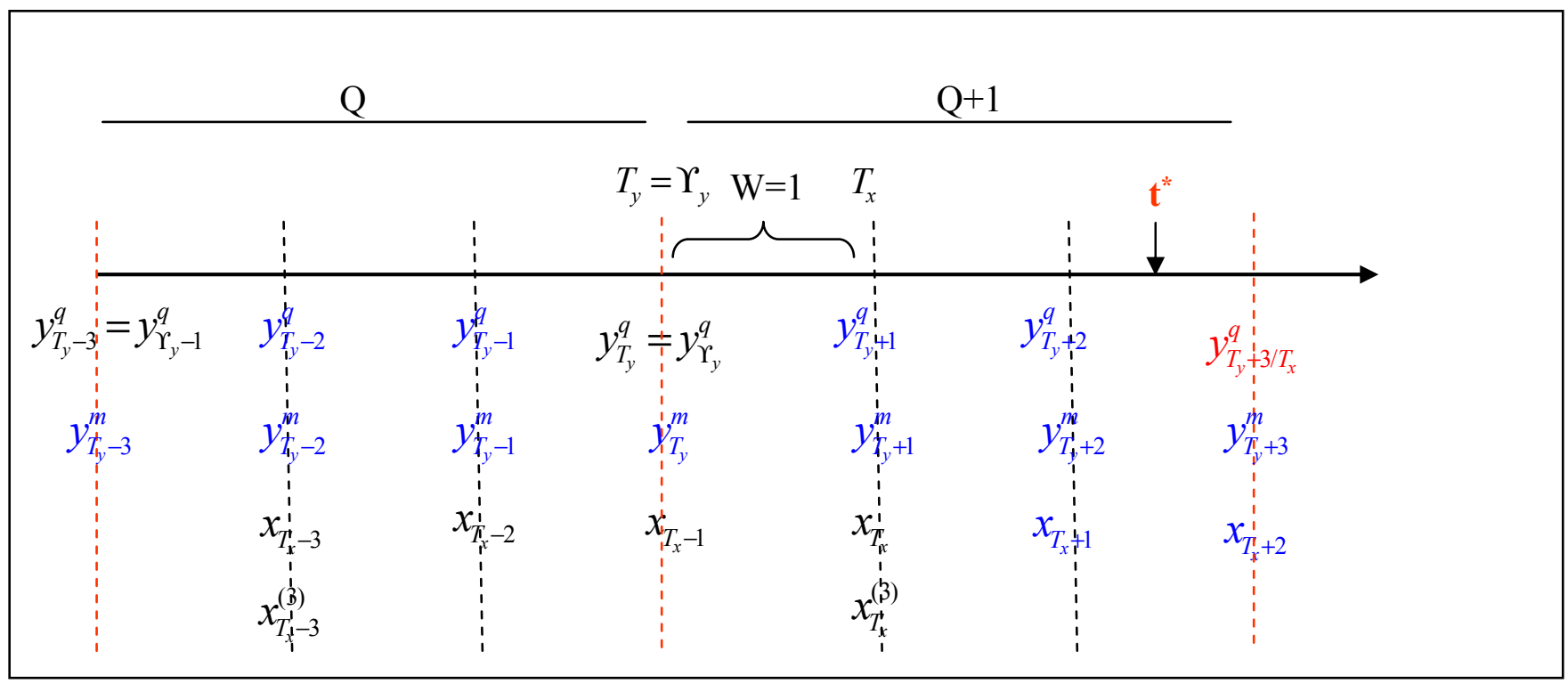

Notes. The timeline shows information available for forecasting the quarterly growth rates of the variable of interest at $T_{y+3}$, which appears in red. This variable is expressed at monthly frequency in the first row so the first two figures of each quarter are not observed. The underlying unobserved monthly growth rates appear in the second row. The monthly growth rates of the indicator appear in the third row and its latest available figure is dated as $T_{x}$. The last row refers to the skip sampled indicator. Observed figures are in black and unobserved figures are in blue. 
Figure 2. Mixing quarterly and monthly observations

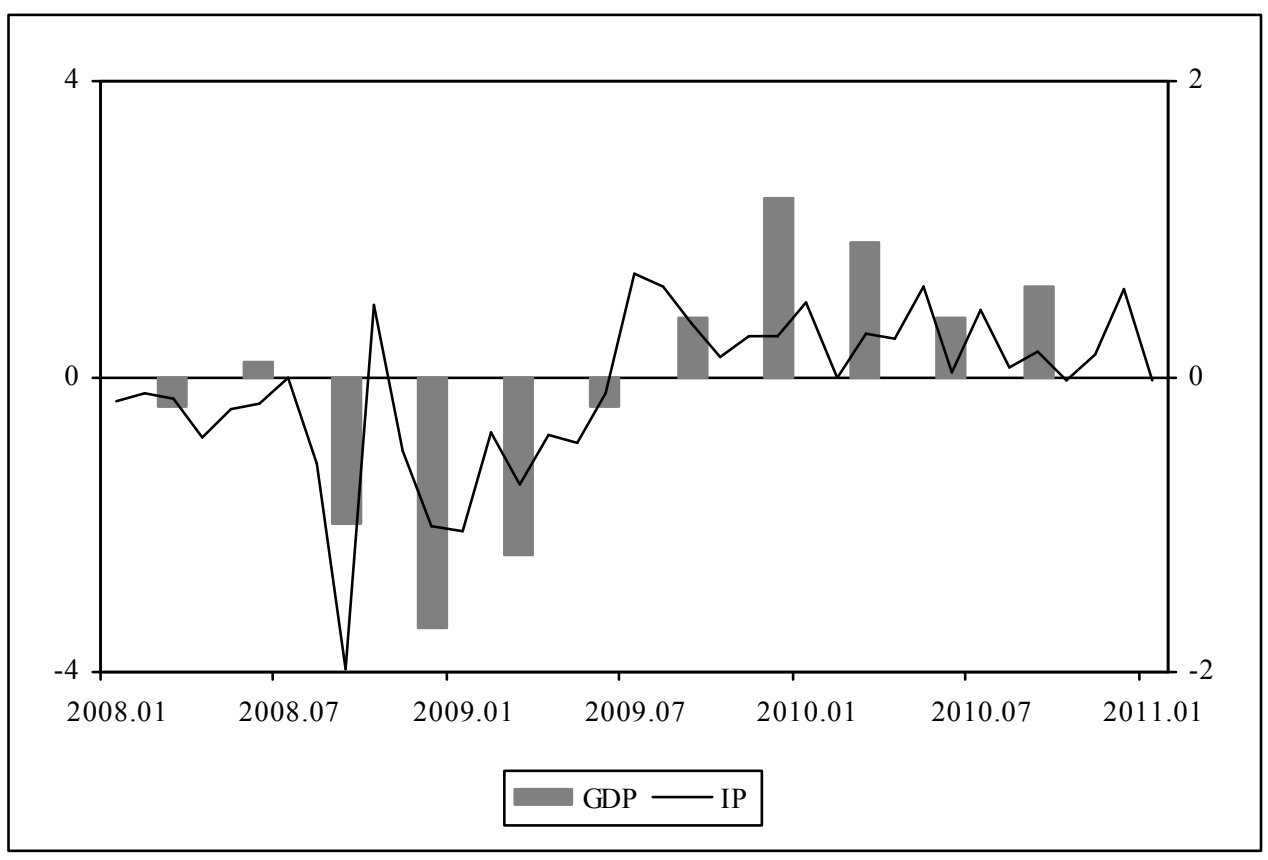

Notes. The line and the bars refers to the latest available time series of industrial production monthly growth rates and GDP quarterly growth rates. 
Figure 3. exponential Almon weighting function

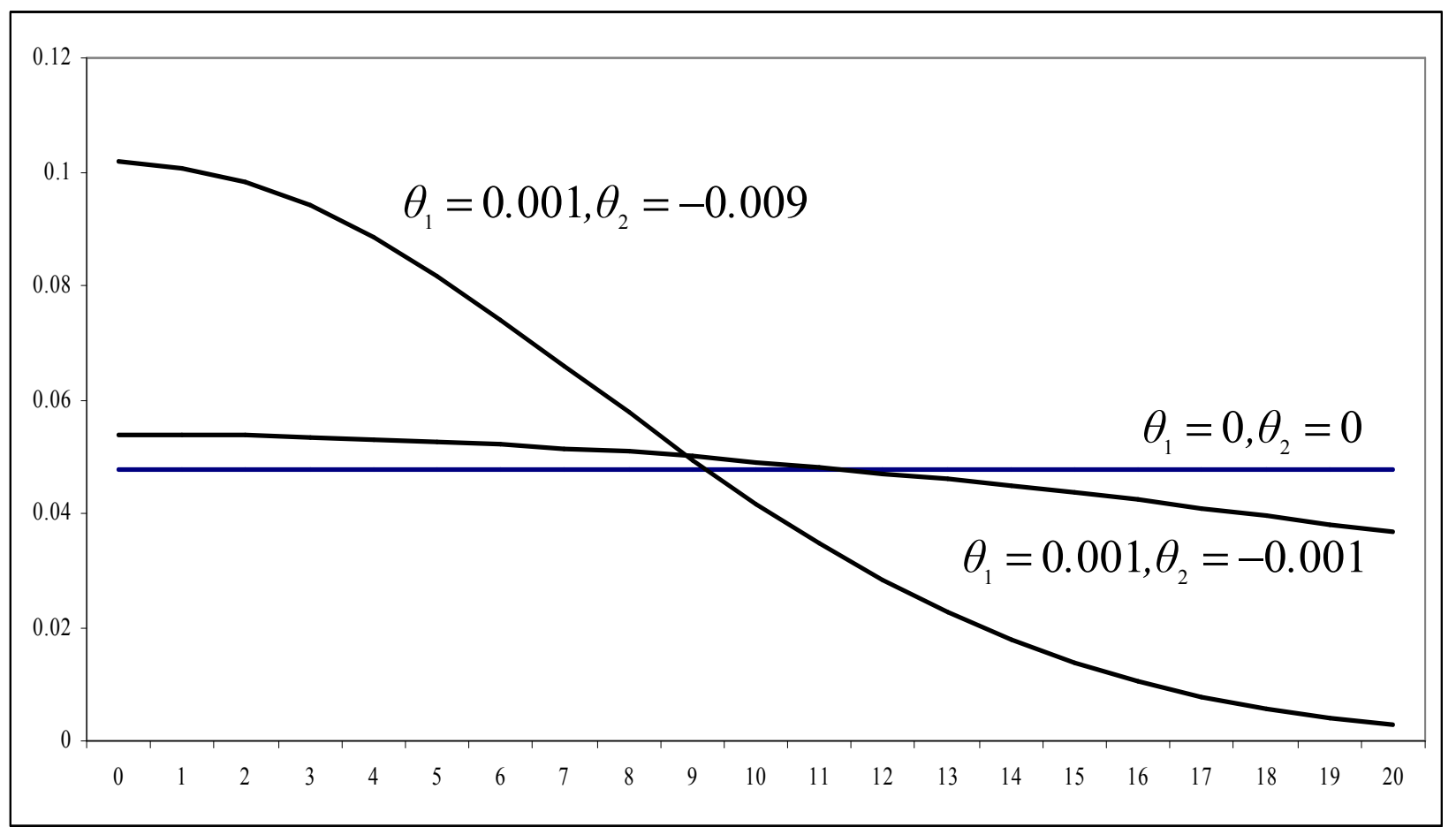

Notes. The figure shows various shapes of the exponential Almon specification whose weights refer to the first 20 lags The shapes are determined by the values of the parameters $\theta_{1}$ and $\theta_{2}$, which are chosen only to illustrate the flexibility of this specification. 
Figure 4. Filtered probabilities of recession

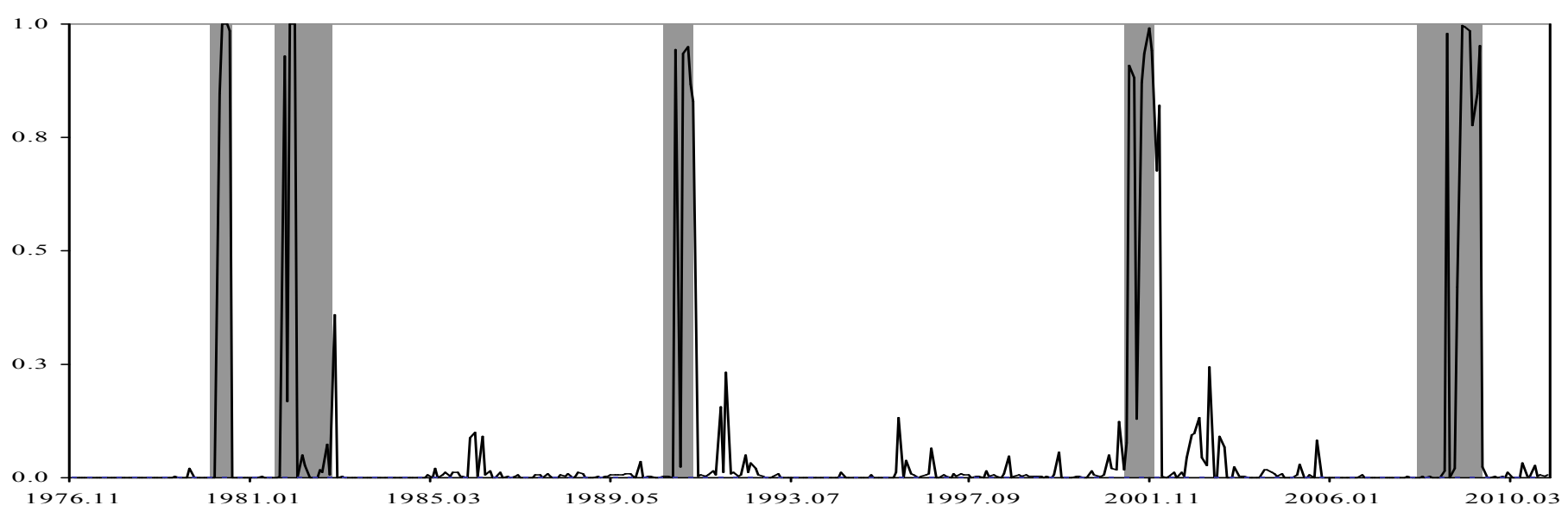

Notes. Shaded areas correspond to the NBER recessions. The figure plots the probabilities of recession in real time in period $t$ using an unbalanced panel with published information in $t$. 